\title{
Numerical modelling of skin tumour tissue with temperature-dependent properties for dynamic thermography
}

\author{
J. Iljaž ${ }^{* 1}$, L. C. Wrobel ${ }^{2,3}$, M. Hriberšek ${ }^{1}$ and J. Marn ${ }^{1}$ \\ 1 - Faculty of Mechanical Engineering, University of Maribor \\ Smetanova 17, SI-2000 Maribor, Slovenia, * corresp: jurij.iljaz@um.si \\ 2 - Brunel University London, Kingston Lane, Uxbridge, UB8 3PH, United Kingdom \\ 3 - Department of Civil and Environmental Engineering, \\ Pontifical Catholic University of Rio de Janeiro (PUC-Rio), \\ Rua Marquês de São Vicente 225, Rio de Janeiro 22451-900, Brazil
}

July 17, 2019

\begin{abstract}
Dynamic thermography has been clinically proven to be a valuable diagnostic technique for skin tumor detection as well as for other medical applications, and shows many advantages over static thermography. Numerical modelling of heat transfer phenomena in biological tissue during dynamic thermography can aid the technique by improving process parameters or by estimating unknown tissue parameters based on measurement data. This paper presents a new non-linear numerical model of multilayer skin tissue containing a skin tumour together with thermoregulation response of the tissue during the cooling-rewarming process of dynamic thermography. The thermoregulation response is modelled by temperature-dependent blood perfusion rate and metabolic heat generation. The aim is to describe bioheat transfer more realistically. The model is based on the Pennes bioheat equation and solved numerically using a subdomain BEM approach treating the problem as axisymmetrical. The paper includes computational tests for Clark II and Clark IV tumours, comparing the models using constant and temperature-dependent properties which showed noticeable differences and highlighted the importance of using a local thermoregulation model. Results also show the advantage of using dynamic thermography for skin tumour screening and detection at an early stage. One of the contributions of this paper is a complete sensitivity analysis of 56 model parameters based on the gradient of the surface temperature difference between tumour and healthy skin. The analysis shows that size of the tumour, blood perfusion rate, thermoregulation coefficient of the tumour, body core temperature and density and specific heat of the skin layers in which the tumour is embedded are important for modelling the problem, and so have to be determined more accurately to reflect realistic skin response of the investigated tissue, while metabolic heat generation and its thermoregulation are not.
\end{abstract}


Keywords: Bio-heat, skin tumour, dynamic thermography, temperature-dependent properties, numerical model, sensitivity analysis

\section{Introduction}

Thermography or infrared thermal (IRT) imaging has become a very valuable tool in recent times due to the development of modern infrared (IR) cameras, electronics and technology and is used mostly for monitoring where heat transfer plays an important role. The IR camera measures the thermal radiation that is being emitted from the observed surface and, based on the intensity and emissivity of the surface, the temperature can be evaluated. Because the method measures the temperature in a contactless manner over the surface, it has an advantage over contact measurement techniques like temperature sensors that have to be in contact with the observed material or media and can measure the temperature only at one point. Therefore, thermography also found practical use in medicine for various applications like breast cancer diagnostics, diagnosis of vascular disorder, fever screening, dental diagnostics, dermatological applications and blood pressure monitoring [1-4].

Thermography for medical screening or diagnosis can be done in two ways, observing the temperature under steady-state condition (passive or static thermography) or inducing thermal stresses by cooling or heating of the observed tissue and measuring the thermal response during the recovery phase (active or dynamic thermography). Both methods have been used for medical applications; however, recent research on dynamic thermography shows a superior approach over the static one due to uncovering more information about the observed tissue, gathered from the dynamic response [5-13]. In this paper, we will focus on the use of dynamic thermography for skin tumour diagnostic. Early detection of the skin tumour is very important for the survival rate of patients, especially in the case of malignant melanoma [14-18]. Skin lesions have different physiological and thermal properties like blood perfusion rate, metabolic heat generation, thermal diffusivity from the healthy skin due to the pathological change of the tissue (vascularisation or angiogenesis) [8, 9, 19-22], which is reflected in different skin temperatures that can be detected using IRT imaging. The temperature difference between the tumour region and the surrounding healthy skin can be small, especially for an early stage lesion under steady-state conditions, where the measurement and background noise produced by the subcutaneous tissues or vessels have a great effect on the thermal image contrast. Therefore, static thermography fails to detect an early stage skin tumour; however, it can be used to detect skin lesions at a later stage, when the temperature contrast is big enough to be detected and is not affected by noise. This drawback is overcome by dynamic thermography with induced thermal stress to the observed tissue, usually cooling, where a much higher temperature difference or contrast during the thermal recovery is produced [8-11], which can be easily detected even with the presence of background noise. Temperature resolution or sensitivity of IR cameras nowadays is in the range of $20-60 \mathrm{mK}$, depending on the quality of the camera, which is expressed by the NETD (Noise Equivalent Temperature Difference) $[2,7,8,12,14]$. The NETD is a measure of how well an IR camera detector is able to distinguish between small differences in thermal radiation. Therefore, it is impossible to measure the temperature contrast between 
tumour and healthy skin that is lower than the NETD, for which an IR camera with low value of NETD is preferable. As stated, dynamic thermography has an advantage over the static one because of the higher temperature contrast obtained between the lesion and surrounding healthy skin during the thermal recovery, and also because of the shorter total examination time of the patient, which is in the range of $2-5 \min [6,8,9,11,14,23]$. The total examination time in static thermography is longer because of the time needed for the patient to acclimatise to the examination room conditions to achieve equilibrium or steady-state condition, and is in the range of $15-60 \mathrm{~min}[5,14,24]$. For these reasons, dynamic thermography shows to be a promising diagnostic technique in dermatology for skin tumour screening, diagnostic and treatment monitoring.

Godoy et al. [8] performed an extensive study on more than 100 patients on the usage of dynamic thermography for skin cancer screening and diagnosis. They used a Ranque-Hilsch vortex tube to cool down the investigated skin lesion for $30 s$ and the observed rewarming period was taken as $2 \mathrm{~min}$. They analysed the captured data with a computer program developed by the authors and compared the results with biopsy results. Among 102 subjects, 59 had benign lesions, 29 had basal-cell carcinoma (BCC), 8 had squamous-cell carcinoma (SCC) and 6 had malignant melanoma (MM). They obtained a sensitivity of $95 \%$ and specificity of $83 \%$, which shows that dynamic thermography can be a promising diagnostic technique. A similar study was done by Çetingül and Herman [14], who carried out clinical tests of dynamic thermography on 35 patients, and proved that inducing thermal stress by cooling of the observed lesion can help distinguish lesions with high blood perfusion or metabolic rate (malignant melanoma or squamous cell carcinoma) from pigmented moles in their early stage (Clark II level). They also used numerical simulation of one of the malignant melanoma trying to reproduce the thermal response during the rewarming period. They concluded that the blood perfusion of the lesion has the biggest influence on predicting the temperature response. In this manner, numerical simulation can be helpful because, from the known temperature response or temperature measurement, it is possible to determine or estimate unknown parameters of the observed tissue or lesion based on an inverse problem. The numerical model used in solving inverse problems plays a significant role and, therefore, it is very important to use a numerical model that describes bioheat transfer during dynamic thermography as realistically as possible.

Numerical models used to model the bioheat transfer in skin for the purpose of laser treatment, estimation of skin or breast tumour parameters, modelling dynamic thermography, are mostly simple taking into account only the tumour and surrounding tissue, and modelling the heat transfer in 2D [25-31]. Focusing only on the modelling of dynamic thermography for skin tumour identification, Cheng and Herman [11] used a simple 2D numerical model taking into account different tissue layers. They numerically investigated different skin cooling approaches for dynamic thermography. Therefore, they solved several direct bioheat problems to determine which parameters (cooling temperature, cooling time, heat transfer coefficient) have the greatest effect on the temperature difference between lesion and surrounding tissue during the thermal recovery. Çetingül and Herman $[10,14]$ used a more realistic 3D model of skin composed by different tissue layers (muscle, fat, epidermis, papillary dermis, reticular dermis and lesion) to model the rewarming process of dynamic thermography. They numerically investigated how the shape of the skin lesion affects the temperature distribution on the skin surface, together with a sensitivity analysis of some model parameters such as thickness, blood perfusion 
rate, thermal conductivity, etc. [10]. They concluded that the actual shape of the lesion had little effect on the temperature distribution and that the most important geometrical parameters are average thickness and diameter (volume) of the lesion. They also concluded that other model parameters do not greatly affect the temperature distribution and that the variation in temperature difference is small. A similar numerical model has also been used by Bhowmik et al. [32-34], who solved inverse bioheat problem of estimating the diameter, thickness, blood perfusion rate and metabolic heat generation of the skin tumour under steady-state conditions (static thermography). They included thermally significant vessels to the skin model under the lesion [32], which affects the lesion identification in its early stage. Later, they improved the possibility of lesion identification over thermally significant vessels using FMTWI (Frequency Modulated Thermal Wave Imaging) [33]. Another similar numerical study has been done by Bonmarin and Gal [7] on lock-in thermal imaging for detection of early-stage melanoma. They used a 3D model similar to Çetingül and Herman [14] and showed that the amplitude and phase image obtained from the periodical thermal stimulation can be of great importance for early detection, especially when the penetration depth of the lesion is less than $0.1 \mathrm{~mm}$. We can conclude that numerical models described in the work of Çetingül and Herman $[10,14]$ or Bhowmik and Repaka [34] are the closest to describe bioheat transfer in skin tumour realistically. However, none of them took into account local thermoregulation of the skin tissue [35-37], which mostly affects the blood perfusion rate. Fiala et al. [35] and others [36, 38-40] modelled the local tissue temperature response using an exponential temperature distribution when simulating thermoregulation of the whole body. Therefore, to successfully model the bioheat transfer during the cooling-rewarming process of dynamic thermography, the local thermoregulation response of the tissue needs to be included in the model.

This paper represents a new approach for modelling dynamic thermography for skin tumour diagnostic by including the local skin and tumour thermoregulation response. The numerical model of the skin tumour tissue presented in this paper will describe the thermal response to the external thermal stimulus more realistically. The problem will be treated as 3D, including different layers of tissue and temperature-dependent properties for blood perfusion rate and metabolic heat generation. It is developed based on the Pennes bioheat model while the subdomain Boundary Element Method (BEM) approach is used to solve the direct bioheat problem efficiently. The paper compares the response of the newly developed model with a standard model using constant properties for Clark II and Clark IV skin tumours, to investigate the difference in these two models. However, the success of accurate modelling of dynamic thermography for skin tumour diagnosis depends not only on the accuracy of the bioheat mathematical model and local thermoregulation but also on the accuracy of the model parameters. Because there is some uncertainty in the model parameters such as the value of material properties, geometry, thickness or thermal response, a sensitivity analysis of 56 parameters has been done on Clark II and Clark IV tumours, to determine which parameters have the strongest correlation with the skin temperature response and, therefore, has to be defined more precisely for accurate numerical simulation of dynamic thermography. The sensitivity analysis is also helpful for inverse bioheat problems of this type, because of the information it provides on which of these parameters could be estimated using dynamic thermography as a measurement technique [12].

The paper is organised as follows: Section 2 presents the numerical model of the 
skin tissue including tumour, governing equation, thermoregulation model and boundary conditions, Section 3 describes the subdomain BEM approach and numerical formulations for solving 3D non-linear bioheat problem, Section 4 discusses the test examples for Clark II and IV tumours, material properties, computational mesh, etc., together with a description of the sensitivity analysis. Section 5 discusses the results obtained with the proposed numerical model using temperature-dependent and constant material properties, together with the results of the sensitivity analysis to determine the most important model parameters. The paper closes with Section 6 that summarises this work and emphasises the importance of its results.

\section{Numerical model}

The numerical model of skin tumour treats skin tissue as non-homogeneous, composed of several different layers as can be seen in Figure 1, representing the computational domain. Its basis has been taken from [10, 11, 14, 34]. Çetingül and Herman [10] concluded that the lesion shape does not affect the surface temperature response and that only the average thickness and diameter or volume is important. Therefore, we decided to model the lesion geometry as a cylinder, with representative diameter and thickness, placed in the centre of our computational domain, as has been done by Bhowmik and Repaka [34]. However, the computational domain or surrounding skin is not modelled as a square [10,34], but as a cylinder because of the applied boundary conditions. Both papers $[10,34]$ used the adiabatic boundary condition on all four sides and, therefore, the computational domain can also be modelled with cylindrical geometry if the boundary is far away from the lesion. In this way, the bioheat transfer problem has been treated as a 3D-axisymmetrical one, reducing computational time. Because of the cylindrical shape of the computational domain and tumour, it is more convenient to treat the problem

a)

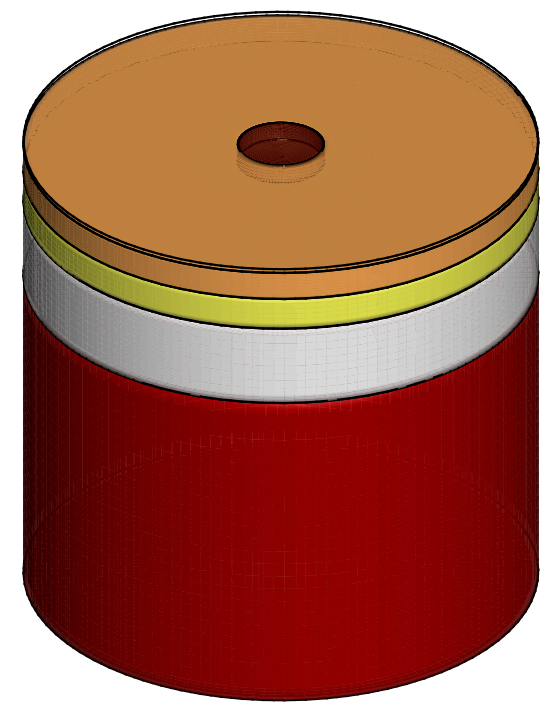

b)

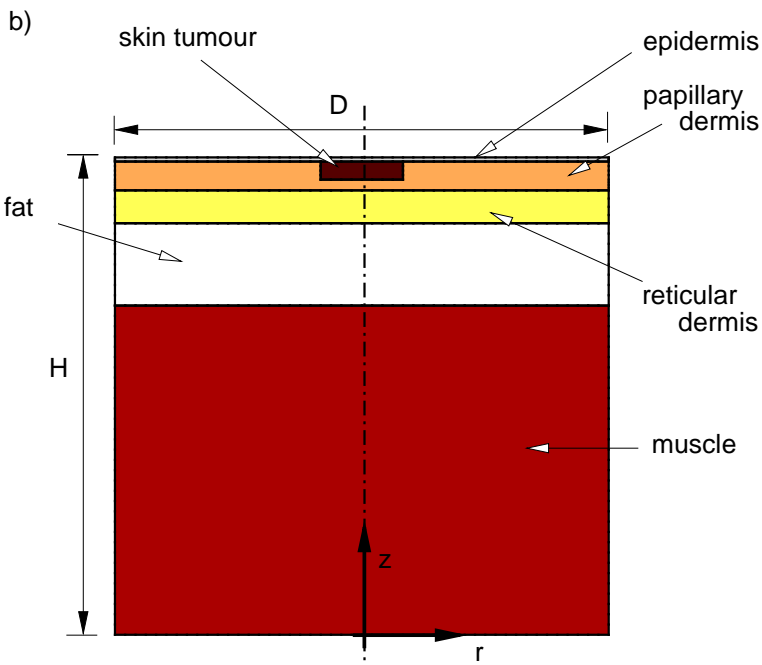

Figure 1: Geometry of numerical model: a) isometric view and b) cross-sectional view with annotation. 
in a cylindrical coordinate system; $\vec{r}=\vec{r}(r, \varphi, z)$, which will be used in this paper to describe the problem. To model the thermal response of the skin more realistically, it is necessary to model the problem as $3 \mathrm{D}$ and not as $2 \mathrm{D}$ or $1 \mathrm{D}$ which can lead to wrong solutions and conclusions. We observed that the temperature response determined with $1 \mathrm{D}$ or $2 \mathrm{D}$ computational models overestimate the temperature difference between lesion and healthy skin.

The model shown in Figure 1 is derived for skin tissue including tumour in a general way. Therefore, it can be applied for investigations in different parts of the body like arm, leg, torso, etc., that have different layer thickness or composition. We can add, subtract or replace the layers with different ones like bone instead of muscle, if we investigate a skin lesion that is close to the bone. However, this model can also be used for simulation of skin thermal response without a lesion.

\subsection{Governing equation}

To describe heat transfer in biological tissue, the Pennes bioheat model has been used because of its wide acceptance and its relative simplicity. There are other more complicated bioheat models that include convection, counter-current and non-Fourier effects, however they are rarely used because of the complexity and unknown model parameters [41-43]. The Pennes mathematical model [44] is generally written as:

$$
\rho c_{p} \frac{\partial T}{\partial t}=\vec{\nabla} \cdot(\lambda \vec{\nabla} T)+\omega_{b} \rho_{b} c_{p, b}\left(T_{a}-T\right)+q_{m},
$$

where $\vec{\nabla}$ represents the nabla operator; $\vec{\nabla}=\left(\frac{\partial}{\partial r}, \frac{\partial}{r \partial \varphi}, \frac{\partial}{\partial z}\right), T$ is tissue temperature, $\rho, \lambda$ and $c_{p}$ are the effective tissue density, thermal conductivity and specific heat, respectively, $\omega_{b}$ is blood perfusion rate, $\rho_{b}$ blood density, $c_{p, b}$ is specific heat of the blood, $T_{a}$ is arterial blood temperature, $t$ time and $q_{m}$ metabolic heat source. The blood perfusion rate is a scalar representing the volumetric blood flow rate per volume of tissue through small arterioles and capillary bed (thermally significant vessels). The blood perfusion acts like a heat source or sink inside tissue, depending on the temperature difference between tissue and arterial blood flow. During the cooling process in dynamic thermography, blood perfusion acts like a heat source, heating up the tissue during thermal recovery, similar to the metabolic heat source which depends on cell activity. Between these two effects, blood perfusion plays a major role in reheating the tissue.

All the parameters in Pennes equation (1) are usually treated as constant. There are many factors that affect the value of these parameters, from chemical reactions to thermoregulation response of the tissue. The parameters are usually evaluated or estimated because of the lack of measurement data or because the values have large deviation $[10,45,46]$. It is known that central and local thermoregulation of the human body influence the metabolic heat generation and blood perfusion rate of the skin, muscle and other tissues, which play an important role in keeping the body core temperature as constant as possible $[35,38,39]$. In other words, thermoregulation tries to keep the human body warm in cold conditions and vice-versa. Therefore, in this paper, because of the cooling of the skin tissue, a local thermoregulation model that describes temperature-dependent blood perfusion and metabolic heat generation will be used; $\omega_{b}=\omega_{b}(T), q_{m}=q_{m}(T)$. Of course, other material properties also depend on the temperature; however, because there 
is no available model to describe the dependency and because the temperature change in cold provocation is relatively small, other material properties will be treated as constant.

Arterial blood temperature $T_{a}$ in Pennes model is usually treated as constant and equal to the body core temperature $[10,14,25-30,34]$. The reason for this is in the definition of blood perfusion rate and assumption that the heat transfer in thermally insignificant vessels (artery, large arterioles and veins) is negligible compared to heat transfer in capillary bed. Therefore, arterial blood flow reaches capillary bed with body core temperature. The change of $T_{a}$ is only reasonable when the thermoregulation of the whole body is modelled [35, 36, 38-40]. In dynamic thermography the application of local cold stress does not affect the body core temperature or the central nervous system, as well as the fact that the patient is acclimatised during the examination and is in a resting position. Therefore, the arterial blood temperature in the numerical model will be treated as constant.

Because the Pennes bioheat equation is written for each layer or tissue in our model, equilibrium and compatibility conditions have to be imposed along the interface between layers to describe the heat transfer in the whole computational domain. The equilibrium and compatibility conditions are:

$$
T_{i}(\vec{r}, t)=T_{i+1}(\vec{r}, t), \quad \lambda_{i} \frac{\partial T_{i}(\vec{r}, t)}{\partial \vec{n}_{i}}=-\lambda_{i+1} \frac{\partial T_{i+1}(\vec{r}, t)}{\partial \vec{n}_{i+1}}
$$

where $\vec{r}=\vec{r}(r, \varphi, z)$ represents the space vector, $\vec{n}=\vec{n}\left(n_{r}, n_{\varphi}, n_{z}\right)$ the normal to the interface boundary, which usually points outside the subdomain, and indices $i$ and $i+1$ represent adjoining subdomains or tissues.

\subsection{Thermoregulation model}

The developed numerical model of skin tumour tissue for simulating dynamic thermography in this paper treats tissue metabolic heat generation and blood perfusion rate as temperature-dependent due to the local thermal stimulus. Modelling local thermoregulation response is still hard due to the lack of accurate numerical models and experimental data.

Fiala et al. [35, 47] and others [38-40, 46, 48] used the van't Hoff $Q_{10}$ effect to model the temperature change of basal metabolic heat generation rate in tissue. Fiala et al. $[35,47]$ also included shivering and exercise effects and wrote the model for metabolic heat source as:

$$
q_{m}=q_{m, b a s}+\Delta q_{m, b a s}+\Delta q_{m, s h}+\Delta q_{m, w},
$$

where $q_{m, b a s}$ represents the basal metabolic rate at rest, $\Delta q_{m, b a s}$ the change of basal metabolic rate because of temperature change, $\Delta q_{m, s h}$ the shivering effect that is controlled by the central nervous system and $\Delta q_{m, w}$ the change in metabolic rate due to work or exercise. For our numerical model the last two terms have been neglected, because the patient is resting during the examination as we are assuming a condition of thermal comfort, and the cooling process does not affect the central nervous system. The basal metabolic change due to temperature is modelled using the $Q_{10}$ effect as [35, 39, 47]:

$$
\Delta q_{m, \text { bas }}=q_{m, \text { bas }}\left(Q_{10, m}^{\left(\frac{T-T_{0}}{10}\right)}-1\right)
$$


where $Q_{10, m}$ represents the metabolic rate coefficient that is usually taken as $2, T$ is local tissue temperature and $T_{0}$ is the equilibrium temperature of the body. The $Q_{10}$ model predicts that the reaction rate of metabolism is doubled when the temperature increases by $10^{\circ} \mathrm{C}$. If we include the model (4) into equation (3), and considering thermal comfort and resting position, the thermoregulation model for metabolic heat generation that has been used in the current numerical model can be written as [39, 40, 46, 48]:

$$
q_{m}(T)=q_{m, b a s} Q_{10, m}^{\left(\frac{T-T_{0}}{10}\right)} .
$$

Thermoregulation of the blood perfusion rate in skin and other tissues has usually been modelled similarly to the metabolic heat generation, because the blood perfusion rate is in correlation with the metabolic rate by oxygen and nutrition demand. Fiala et al. [35, 47] used the proportional model of the metabolic rate change, while Laszczyk and Nowak [40] and Silva et al. [38] used the same model as for the metabolic rate:

$$
\omega_{b}(T)=\omega_{b, b a s} Q_{10, b}^{\left(\frac{T-T_{0}}{10}\right)},
$$

where now $Q_{10, b}$ represents the blood perfusion rate coefficient, which is usually the same as $Q_{10, m}$, and $\omega_{b, b a s}$ represents the basal blood perfusion rate. A similar model for skin and tissue blood perfusion rate has also been used by Stolwijk [49] and others [50-52] using different values for the denominator (from 4 to 7 ), which affects only the rate change. The denominator for the proposed model has been chosen as 10 [35, 38, 47, 53], while the blood perfusion rate or metabolic heat generation rate change can be controlled by the value of the $Q_{10}$ coefficient.

It is difficult to exactly model the thermoregulation response of blood perfusion rate due to local vascular dilation or contraction because of the lack of validated models, especially for tumour tissue that has a different vascular system $[54,55]$ and reacts to temperature changes differently [21, 22]. Song et al. [21, 22] did not observe any significant change in blood perfusion rate for tumour during hyperthermia, compared to the skin or muscle tissue. The blood perfusion rate for tumour can slightly increase by increasing the temperature up to $42^{\circ} \mathrm{C}$ and then decreases drastically by increasing the temperature even further, while the skin and muscle tissue show exponential change up to ten times compared to the normal level. Some authors used this correlation for skin, muscle, fat and tumour tissue to model the tissue response during hypothermia treatment $[56,57]$, while Sun et al. [58] used a linear approximation for the blood perfusion rate of tumour during cooling and heating treatment. From this review, we are assuming that the temperature change of the blood perfusion rate for tumour is not as distinctive as for skin or muscle tissue, and can be treated with nearly constant response. The justification for this mild temperature response of the tumour blood perfusion rate can be found in the dense vascular system. The blood perfusion rate for skin tumour is already much higher compared to healthy skin, from 2 times for BCC (basal cell carcinoma) to 5.5 times for malignant melanoma [20] and is therefore not affected by temperature change as much as the healthy tissue. However, to model the thermal response of skin tumour more accurately, more precise measurements are needed, especially for the cooling response.

In this study we model metabolic heat generation and blood perfusion rate of the tissue as temperature-dependent with models (5) and (6). However, to take into account the different behavior of tumour, we change the value of the coefficient $Q_{10}$. Figure 
2 shows how the value of coefficient $Q_{10}$ affects the relative change of parameter with temperature. We can observe an exponential behavior for a value of 2 , while for values near 1 the behaviour is almost linear, and it is constant for a value of 1 .

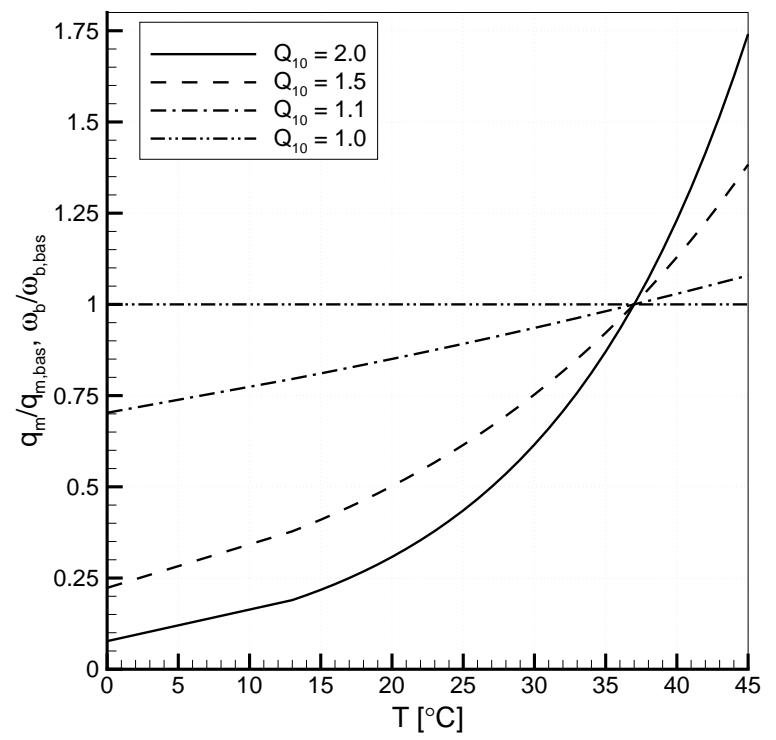

Figure 2: Relative change of metabolic heat source $\left(q_{m} / q_{m, b a s}\right)$ and blood perfusion rate $\left(\omega_{b} / \omega_{b, b a s}\right)$ with the $Q_{10}$ coefficient; $T_{0}=37^{\circ} \mathrm{C}$.

\subsection{Boundary conditions}

To simulate dynamic thermography we also have to prescribe appropriate initial and boundary conditions for the computational domain that, due to the axisymmetrical treatment of the problem, do not depend on the angle $\varphi$.

For the bottom part, we prescribe the Dirichlet boundary condition assuming that the muscle tissue is thick enough to reach the body core temperature, which does not change with time:

$$
T(r, z, t)=T_{0}, \quad z=0, \quad 0 \leq r \leq D / 2, \quad 0 \leq t \leq \tau,
$$

where $T_{0}$ represents the temperature of the body that is in thermal equilibrium with the surrounding environment (body core temperature), $D$ is the diameter of the computational domain and $\tau$ represents the simulation time including the cooling process.

On the sides of the computational domain, we prescribe adiabatic boundary condition assuming that the diameter $D$ of the computational domain is large enough not to affect the numerical solution:

$$
\frac{\partial T}{\partial r}(r, z, t)=0, \quad 0 \leq z \leq H, \quad r=D / 2, \quad 0 \leq t \leq \tau,
$$

where $H$ represents the total height of the computational domain.

For the skin surface, we have to take into account the cooling process as well as the heat transfer with the surrounding environment during the rewarming period. Çetingül 
and Herman [10, 14] and Bhowmik and Repaka [34] used a constant temperature cooling approach, while Cheng and Herman [11] numerically tested different cooling approaches using constant temperature cooling, cooling with a cotton patch soaked in water and convection cooling. The constant cooling approach seems to be the most appropriate because of the deep penetration and high temperature contrast during the rewarming period. Strąkowska et al. [12] also used a constant temperature cooling approach with a Peltier cooling device for their experiment. Therefore, for the numerical model presented in this paper, we used a constant cooling approach, which can be written as:

$$
T(r, z, t)=T_{c}, \quad z=H, \quad 0 \leq r \leq D / 2, \quad 0 \leq t \leq t_{c}
$$

where $T_{c}$ represents the cooling temperature and $t_{c}$ the cooling time period. Here, an assumption of good contact between the cooling element and the skin is made, not including the contact resistance that can affect the thermal contrast during the rewarming process. After the cooling period the skin is exposed to the surrounding environment, which can be described with the Robin boundary condition:

$$
\lambda \frac{\partial T}{\partial z}(r, z, t)=\alpha\left(T(r, z, t)-T_{\infty}\right), \quad z=H, \quad 0 \leq r \leq D / 2, \quad t_{c}<t \leq \tau,
$$

where $\alpha$ represents the heat transfer coefficient, $T$ local temperature of the tissue and $T_{\infty}$ surrounding temperature. The heat transfer coefficient can include many effects such as heat convection, thermal radiation and water evaporation [34]. However, thermal radiation is negligible in this case due to the small temperature difference between the skin and surrounding environment and does not affect the rewarming process, as well as the water evaporation by sweating $[11,14]$. Sweating is important only when the body temperature increases due to exercise, work, environmental conditions, etc., or increased skin temperature $[59,60]$, which is not the case for dynamic thermography. The main contribution during rewarming is therefore heat convection with the surrounding environment, which is not intense and the skin rewarms mostly because of the internally generated heat or blood perfusion. The assumption for using the described boundary conditions for the computational domain (8) and cooling (9) is that the diameter of the cooling element is larger than the computational domain; $D_{c}>D$. Otherwise, we would have to prescribe different boundary conditions at the skin surface, one for the cooling element and one for the surrounding skin.

For the initial temperature condition $T(r, z, t=0)$, we prescribed the steady-state solution of the bioheat problem determined with the boundary conditions (7), (8) and (10).

\section{Boundary Element Method}

To solve the numerical model described in this paper, the subdomain BEM approach has been used. The main reason for choosing this method is its advanced treatment of boundary conditions, where Neumann boundary conditions are directly incorporated in the formulation without any additional approximation, which increases the accuracy of the numerical solution while the subdomain approach increases the computational speed. The subdomain approach has been discussed in detail by Ramšak and Škerget [61, 62], Ravnik et al. [63, 64], as well as in our previous work [65] and will be omitted in this 
paper. We already derived a BEM numerical scheme for solving $2 \mathrm{D}$ transient bioheat problems based on elliptic and parabolic fundamental solutions with great success [65]. Because in this paper we are dealing with 3D axisymmetrical geometry and a non-linear governing equation due to temperature-dependent blood perfusion rate and metabolic heat generation, the derivation of the BEM numerical scheme for solving this type of problems based on the axisymmetrical elliptic fundamental solution will be presented in this section.

The governing equation (1) written for each tissue has been treated in the form of a Poisson equation:

$$
\vec{\nabla}^{2} u(\vec{r})=b(\vec{r}),
$$

where $\vec{\nabla}^{2}$ represents the Laplace operator, $u(\vec{r})$ is an arbitrary field function, $b(\vec{r})$ is the source term or the non-homogeneous part of the equation and $\vec{r}=\vec{r}(x, y, z)$ is an arbitrary spatial vector. We start with the integral form of Green's second identity, which for the Poisson equation (11) is written in the form:

$$
c(\vec{\xi}) u(\vec{\xi})=\int_{\Gamma} \vec{q}(\vec{R}) u^{*}(\vec{\xi}, \vec{R}) d \vec{\Gamma}-\int_{\Gamma} u(\vec{R}) \overrightarrow{q^{*}}(\vec{\xi}, \vec{R}) d \vec{\Gamma}-\int_{\Omega} b(\vec{r}) u^{*}(\vec{\xi}, \vec{r}) d \Omega,
$$

where $\Omega$ and $\Gamma$ represent the geometry domain and boundary, respectively, $\vec{R}=\vec{R}(x, y, z)$ is the spatial vector of the boundary, $\vec{q}=\partial u / \partial \vec{n}$ is the normal derivative of the field function $u, \vec{\xi}=\vec{\xi}(x, y, z)$ represents the position of the source point, $c(\vec{\xi})$ the free coefficient that depends on the position of the source point, and $u^{*}(\vec{\xi}, \vec{R})$ and $\overrightarrow{q^{*}}(\vec{\xi}, \vec{R})=$ $\partial u^{*}(\vec{\xi}, \vec{R}) / \partial \vec{n}$ are the fundamental solution and its normal derivative, respectively.

Because the problem has been treated as axisymmetrical, the cylindrical coordinate system $\vec{r}=\vec{r}(r, \varphi, z)$ is introduced:

$$
x=r \cos (\varphi), \quad y=r \sin (\varphi), \quad z=z,
$$

where $r$ represents the radial distance, $\varphi$ the angle between the $x$-axis and the radial distance and $z$ the $z$-axis. The elementary volume and surface can now be written in the cylindrical coordinate system as:

$$
d \Omega=|J| d r d \varphi d z, \quad d \vec{\Gamma}=\vec{n}|J| d \ell d \varphi,
$$

where $|J|=r_{p}$ represents the determinant of the Jacobian matrix, which is equal to the radial distance of an arbitrary point and $d \ell=\sqrt{d r^{2}+d z^{2}}$ the elementary distance. In this manner, the integral equation (12) can be rewritten as:

$$
\begin{gathered}
c(\vec{\xi}) u(\vec{\xi})=\int_{\Gamma} q(\vec{R}) u^{*}(\vec{\xi}, \vec{R}) r_{p} d \ell d \varphi- \\
\int_{\Gamma} u(\vec{R}) q^{*}(\vec{\xi}, \vec{R}) r_{p} d \ell d \varphi-\int_{\Omega} b(\vec{r}) u^{*}(\vec{\xi}, \vec{r}) r_{p} d r d \varphi d z,
\end{gathered}
$$

where $q(\vec{R})=\vec{q}(\vec{R}) \cdot \vec{n}$ and $q^{*}(\vec{\xi}, \vec{R})=\overrightarrow{q^{*}}(\vec{\xi}, \vec{R}) \cdot \vec{n}$. Because of the axisymmetrical treatment of the problem $u(r, \varphi, z)=u(r, z)$, equation (15) can be integrated along the angle $\varphi$ as:

$$
\begin{gathered}
c(\vec{\xi}) u(\vec{\xi})=\int_{\ell} \int_{0}^{2 \pi} q(\vec{R}) u^{*}(\vec{\xi}, \vec{R}) r_{p} d \ell d \varphi- \\
\int_{\ell} \int_{0}^{2 \pi} u(\vec{R}) q^{*}(\vec{\xi}, \vec{R}) r_{p} d \ell d \varphi-\int_{\Pi} \int_{0}^{2 \pi} b(\vec{r}) u^{*}(\vec{\xi}, \vec{r}) r_{p} d \Pi d \varphi
\end{gathered}
$$




$$
\begin{gathered}
c(\vec{\xi}) u(\vec{\xi})=\int_{\ell} q(\vec{R}) u_{a x i}^{*}(\vec{\xi}, \vec{R}) r_{p} d \ell- \\
\int_{\ell} u(\vec{R}) q_{a x i}^{*}(\vec{\xi}, \vec{R}) r_{p} d \ell-\int_{\Pi} b(\vec{r}) u_{a x i}^{*}(\vec{\xi}, \vec{r}) r_{p} d \Pi,
\end{gathered}
$$

where $\Pi$ represents the cross-sectional surface of the domain around the axis of revolution, $d \Pi=d r d z$ its elementary surface and $u_{a x i}^{*}(\vec{\xi}, \vec{r})$ and $q_{a x i}^{*}(\vec{\xi}, \vec{r})$ are the axisymmetrical elliptical fundamental solution and its normal derivative, respectively. The axisymmetrical fundamental solution is given by the equation:

$$
u_{a x i}^{*}(\vec{\xi}, \vec{r})=\frac{K(m)}{\pi(a+b)^{1 / 2}},
$$

where $a, b$ and $m$ represent the parameters that reflect the distance between the source and field points, and $K(m)$ is the complete elliptic integral of the first kind [66, 67]. The parameters $a, b$ and $m$ are:

$$
a=r_{\xi}^{2}+r_{p}^{2}+\left(z_{\xi}-z_{p}\right)^{2}, \quad b=2 r_{\xi} r_{p}, \quad m=2 b /(a+b) .
$$

where the source point is now defined by coordinates $\vec{\xi}=\vec{\xi}\left(r_{\xi}, z_{\xi}\right)$ and the field point by $\vec{r}=\vec{r}\left(r_{p}, z_{p}\right)$, while the normal derivative of the fundamental solution is defined by the equation:

$$
\begin{gathered}
q_{a x i}^{*}(\vec{\xi}, \vec{R})=\frac{1}{\pi(a+b)^{1 / 2}} \frac{1}{2 r_{p}}\left(\frac{r_{\xi}^{2}-r_{p}^{2}+\left(z_{\xi}-z_{p}\right)^{2}}{a-b} E(m)-K(m)\right) n_{r}(\vec{R}) \\
+\frac{1}{\pi(a+b)^{1 / 2}} \frac{z_{\xi}-z_{p}}{a-b} E(m) n_{z}(\vec{R})
\end{gathered}
$$

where $n_{r}(\vec{R})$ and $n_{z}(\vec{R})$ represent the components of the normal vector on the boundary $\vec{n}(\vec{R})=\left\{n_{r}(\vec{R}), n_{z}(\vec{R})\right\}$, and $E(m)$ is the complete elliptic integral of the second kind $[66,67]$.

The value of the free coefficient $c(\vec{\xi})$ depends on the position of the source point $\vec{\xi}$ and is defined as:

$$
\begin{array}{ll}
c(\xi)=1, & \vec{\xi} \in \Pi, \\
c(\xi)=\beta /(2 \pi), & \vec{\xi} \in \ell .
\end{array}
$$

where $\beta$ represents the external angle of the boundary at point $\vec{\xi}$.

As can be seen from equation (17) we managed to reduce the problem dimensions by one using the axisymmetrical approximation, which means that we need to evaluate only surface and line integrals. Figure 3 shows the old and new computational domains. The advantage of this treatment is that we only need to discretise the cross-sectional surface $\Pi$ and boundary $\ell$, which means a much lower number of elements or nodes compared to a full 3D computational mesh. For geometry discretisation four-node linear cells have been used, and consequently two-node linear elements for the boundary. For the approximation of the field function $u(\vec{r})$ and non-homogeneous part $b(\vec{r})$, quadratic interpolation functions have been used and constant interpolation for the normal derivative on the boundary $q(\vec{R})=\partial u(\vec{R}) / \partial n$. The representative element used in the subdomain approach is also shown in Figure 3, together with the position of the interpolation nodes. 

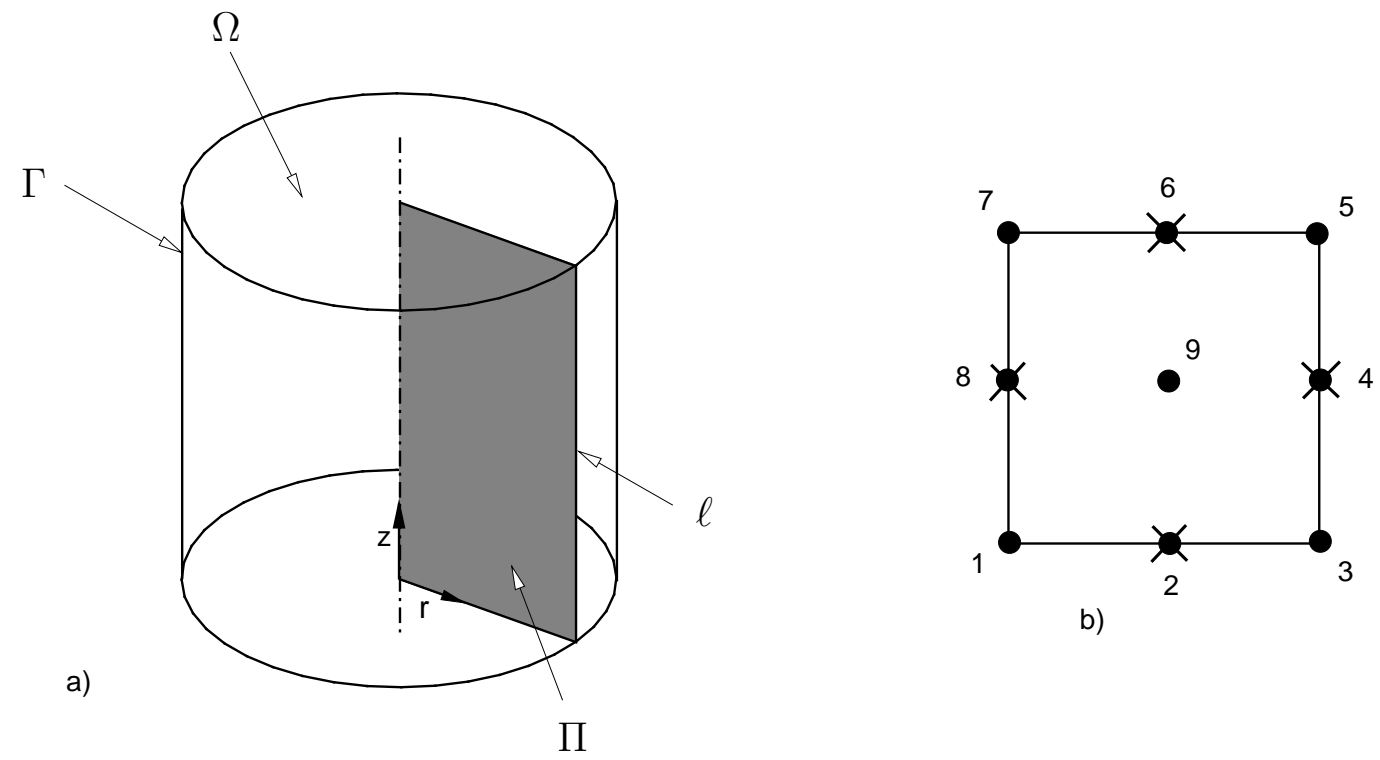

b)

Figure 3: Representation of the computational domain for axisymmetrical problems a) and element type used in BEM b) (black dots represent computational nodes for $T$ and crosses for the normal derivative $q$ ).

Applying expression (17) at every computational node of each cell separately, we obtain a global system of equations for the Poisson equation (11) which can be written as:

$$
[H]\{u\}=[G]\{q\}-[S]\{b\} .
$$

where $[H],[G]$ and $[S]$ are the matrices, $\{u\}$ is the vector of discrete values of the field function, $\{q\}$ is the vector of discrete values of the normal derivative of $u$ and $\{b\}$ is the vector of discrete values of the non-homogeneous part. A more detailed description of the matrix coefficients can be found in our previous work [65].

We can now apply the above procedure to the bio-heat equation (1), where at first we rewrite the equation in the form of the Poisson equation (11):

$$
\vec{\nabla}^{2} T=\frac{1}{a_{d i f f}} \frac{\partial T}{\partial t}-\frac{\omega_{b}(T) \rho_{b} c_{p, b}}{\lambda}\left(T_{a}-T\right)-\frac{q_{m}(T)}{\lambda},
$$

where $a_{d i f f}=\lambda / \rho c_{p}$ represents thermal diffusivity and $\omega_{b}(T)$ and $q_{m}(T)$ are temperaturedependent parameters. The non-homogeneous part $b(\vec{r})$ is now:

$$
b(\vec{r})=\frac{1}{a_{d i f f}} \frac{\partial T}{\partial t}-\frac{\omega_{b}(T) \rho_{b} c_{p, b}}{\lambda}\left(T_{a}-T\right)-\frac{q_{m}(T)}{\lambda} .
$$

The temperature time derivative is estimated by the second-order finite diference (FD) scheme as [65]:

$$
\frac{\partial T}{\partial t} \approx \frac{3 T^{t}-4 T^{t-1}+T^{t-2}}{2 \Delta t}
$$

where superscript indices $t, t-1$ and $t-2$ represent different time steps and $\Delta t$ is the time difference between two adjacent time steps. For the first time step, the following assumption has been made; $T^{t-1}=T^{t-2}$, which reduces the FD time marching scheme to first-order. Including the approximation (25) into equation (23) and using a fully implicit 
scheme $\left(T=T^{t}, q=q^{t}\right)$, the global system of equations (22) can be rewritten as:

$$
\begin{gathered}
\left([H]+\left\{\frac{3}{2 a_{d i f f} \Delta t}+\frac{\omega_{b}\left(T^{t}\right) \rho_{b} c_{p, b}}{\lambda}\right\}[S]\right)\left\{T^{t}\right\}=[G]\left\{q^{t}\right\}+\left\{\frac{2}{a_{\text {diff }} \Delta t}\right\}[S]\left\{T^{t-1}\right\} \\
-\left\{\frac{1}{2 a_{\text {diff }} \Delta t}\right\}[S]\left\{T^{t-2}\right\}+[S]\left\{\frac{\omega_{b}\left(T^{t}\right) \rho_{b} c_{p, b}}{\lambda} T_{a}+\frac{q_{m}\left(T^{t}\right)}{\lambda}\right\} .
\end{gathered}
$$

The system of equations (26) is non-linear and has to be solved using a non-linear iteration loop within each time step. Therefore, the final form of equation (26) is

$$
\begin{aligned}
([H]+ & \left.\left\{\frac{3}{2 a_{d i f f} \Delta t}+\frac{\omega_{b}\left(T_{k-1}^{t}\right) \rho_{b} c_{p, b}}{\lambda}\right\}[S]\right)\left\{T_{k}^{t}\right\}=[G]\left\{q^{t}\right\}+\left\{\frac{2}{a_{\text {diff }} \Delta t}\right\}[S]\left\{T^{t-1}\right\} \\
& -\left\{\frac{1}{2 a_{\text {diff }} \Delta t}\right\}[S]\left\{T^{t-2}\right\}+[S]\left\{\frac{\omega_{b}\left(T_{k-1}^{t}\right) \rho_{b} c_{p, b}}{\lambda} T_{a}+\frac{q_{m}\left(T_{k-1}^{t}\right)}{\lambda}\right\} .
\end{aligned}
$$

where indices $k$ and $k-1$ represent the current and previous non-linear iteration step, respectively. The non-linear loop is controlled by the maximum number of steps and the RMS (Root Mean Square) error as:

$$
k>k_{\max }, \quad \varepsilon_{R M S, k} \leq \varepsilon_{\max },
$$

where $k_{\max }$ represents the maximum number of non-linear steps, $\varepsilon_{R M S}$ the RMS error and $\varepsilon_{\max }$ the maximum error. The RMS error is calculated at every iteration step as:

$$
\varepsilon_{R M S, k}=\sqrt{\frac{1}{n} \sum_{i=1}^{n} \frac{\left(T_{i, k-1}-T_{i, k}\right)^{2}}{T_{i, k}^{2}}},
$$

where index $i$ represents the computational node and $n$ the total number of nodes in the computational domain. When convergence of the non-linear loop is achieved, meaning the RMS error is lower than the prescribed maximum or the maximum number of steps is reached, the non-linear iteration is stopped and the calculation for a new time step can begin.

The non-linear system of equations (27) represents the BEM numerical scheme for solving transient bioheat problems with temperature-dependent blood perfusion and metabolic heat generation. By applying the boundary conditions (7)-(10), the system of equations (27) can be rewritten as a system of linear equations and solved using a standard solver. The result is the temperature field for the whole computational domain and the normal derivative of the temperature on the boundary. From our previous experience [65-67], we find this approach suitable for solving complicated non-linear problems achieving good numerical accuracy and fast computational time.

\section{Computational examples}

The computational tests of dynamic thermography for skin tumour diagnostic presented in this paper are based on the work of Bhowmik and Repaka [34] and Çetingül and Herman $[10,14]$. Unfortunately, these authors treated the problem in a different manner, using 
different boundary conditions, size of tumour, different results presentation, and therefore a direct comparison is not feasible. The numerical model has already been described in detail in section 2. Here, only the chosen value of parameters, layer thickness, tumour dimension, cooling temperature and time step are presented.

The numerical results will be compared for two different tumour sizes, Clark II and Clark IV, showing the difference in thermal response during the rewarming period. For Clark II, the diameter and thickness are $d_{t}=2 \mathrm{~mm}$ and $h_{t}=0.44 \mathrm{~mm}[14,34]$, while for Clark IV, they are $d_{t}=2.5 \mathrm{~mm}$ and $h_{t}=1.1 \mathrm{~mm}$ [34]. The reason for choosing only two different stages, an early stage Clark II and a later stage Clark IV tumour, is to show the difference in thermal contrast obtained by dynamic thermography, as well as how the thermoregulation response affects the temperature contrast. From a diagnostic perspective it is desired to identify the skin lesion in its early stage to improve the survival rate $[9,14,15,19,68]$. We gathered the material properties and thicknesses for the different layers in Table 1 , taken from [10, 14, 34]. The values of $Q_{10, m}$ and $Q_{10, b}$ used in the temperature-dependent metabolic and blood perfusion rate model have been determined based on the work of Fiala et al. [35] and Silva et al. [38]. For all layers, with the exception of the tumour, the value has been set to $Q_{10, m}=Q_{10, b}=2.0$, while for the tumour, we assumed the value based on the literature review of $Q_{10, m}=Q_{10, b}=1.1$.

The mean body core temperature for a healthy person in a resting position is in the range of $36.5^{\circ} \mathrm{C}-37.5^{\circ} \mathrm{C}$, therefore the temperature $T_{0}$ for the boundary condition (7), as well as for the thermoregulation models, has been set to $T_{0}=37.0^{\circ} C[10,14,34]$. The arterial blood temperature has also been taken equal to the mean body core temperature; $T_{a}=T_{0}=37.0^{\circ} \mathrm{C}$, and assumed to be constant during the dynamic thermography test. As for the surrounding conditions, the ambient temperature has been chosen to be $T_{\infty}=22.4^{\circ} \mathrm{C}[14,34]$ and the heat transfer coefficient $\alpha=10 \mathrm{~W} / \mathrm{m}^{2} \mathrm{~K}[11,14]$.

Different authors used different values of the cooling duration $(4 s, 10 s, 60 s$ and $120 s)$ as well as the cooling temperature $\left(4^{\circ} \mathrm{C}, 13^{\circ} \mathrm{C}, 20^{\circ} \mathrm{C}\right.$ and $\left.26^{\circ} \mathrm{C}\right)[10-12,14,34]$. In this paper, the cooling temperature and duration have been taken from Bhowmik and Repaka [34] and Çetingül and Herman [14] and are $t_{c}=60 \mathrm{~s}$ and $T_{c}=13^{\circ} \mathrm{C}$. For the simulation time, we simulate an extra $10 \mathrm{~min}=600 \mathrm{~s}$ of rewarming period, which is long enough. The largest temperature difference between the tumour and surrounding tissue appears up to $60 \mathrm{~s}$ after the end of cooling process and then decline towards a steady-state condition [11]. Therefore, the total simulation time has been set to $\tau=660 \mathrm{~s}$.

\begin{tabular}{c|cc|ccccc} 
Material & $d[\mathrm{~mm}]$ & $h[\mathrm{~mm}]$ & $\rho\left[\mathrm{kg} / \mathrm{m}^{3}\right]$ & $c_{p}[\mathrm{~J} / \mathrm{kgk}]$ & $\lambda[W / \mathrm{mK}]$ & $\omega_{b, \text { bas }}[1 / \mathrm{s}]$ & $q_{m, \text { bas }}\left[\mathrm{W} / \mathrm{m}^{3}\right]$ \\
\hline \hline epidermis & - & 0.1 & 1200 & 3589 & 0.235 & 0.0 & 0.0 \\
papillary dermis & - & 0.7 & 1200 & 3300 & 0.445 & 0.0002 & 368.1 \\
reticular dermis & - & 0.8 & 1200 & 3300 & 0.445 & 0.0013 & 368.1 \\
fat & - & 2.0 & 1000 & 2674 & 0.185 & 0.0001 & 368.3 \\
muscle & - & 8.0 & 1085 & 3800 & 0.510 & 0.0027 & 684.2 \\
\hline tumour Clark II & 2.0 & 0.44 & 1030 & 3852 & 0.558 & 0.0063 & 3680 \\
tumour Clark IV & 2.5 & 1.1 & 1030 & 3852 & 0.558 & 0.0063 & 3680 \\
\hline blood & - & - & 1060 & 3770 & - & - & -
\end{tabular}

Table 1: Material properties and tissue dimensions [10, 11, 14, 34]. 
To assure numerical accuracy of the results, we carried out a mesh and time discretisation analysis, changing the time step and representative element size. We found a similar conclusion to our previous works $[65,69]$. The appropriate element size is $\Delta r=0.5 \mathrm{~mm}$ due to the use of a quadratic interpolation function, and the time step comparing the temperature profiles on the skin surface should be $\Delta t=1 \mathrm{~s}$. Figure 4 shows the structured mesh of the cross-sectional area of the computational domain used for the numerical simulations, where the number of elements in the thin layers was a minimum of two. The domain size used in $[10,14,34]$ was $12 \mathrm{~mm}$, but we found it appropriate to use a larger domain. For a tumour size of $2 \mathrm{~mm}$ in diameter, the surrounding domain size should be at least $22 \mathrm{~mm}$ and for a tumour size $4 \mathrm{~mm}$, at least $25 \mathrm{~mm}$. For this reason, we decided to used a domain size of $D=25 \mathrm{~mm}$ in this study. For the solver, we set a maximum number of non-linear steps to $k_{\max }=10$ and the maximum error to $\varepsilon_{\max }=1 \cdot 10^{-10}$.

\subsection{Sensitivity analysis}

The numerical model presented in this paper has many parameters that are not determined accurately and can vary greatly depending on the person and position on the body (thickness, material properties, etc.) [14, 34]. Therefore, it is difficult to simulate realistic thermal response of dynamic thermography for skin tumour diagnostic if the parameters are not determined accurately. For this reason, the paper also presents a sensitivity analysis of model parameters to determine the most important, that have to be determined as accurately as possible.

Çetingül and Herman [10] carried out a sensitivity analysis for certain model parameters, however not a complete one. They varied the material properties and thickness of the layers in the range of lower and upper bound values found in the literature. However, the relative change of the parameters was not equal for all, and they compare the change of absolute mean surface temperature, which can lead to wrong conclusions. They concluded that the skin thickness and blood perfusion rate have the biggest effect on the
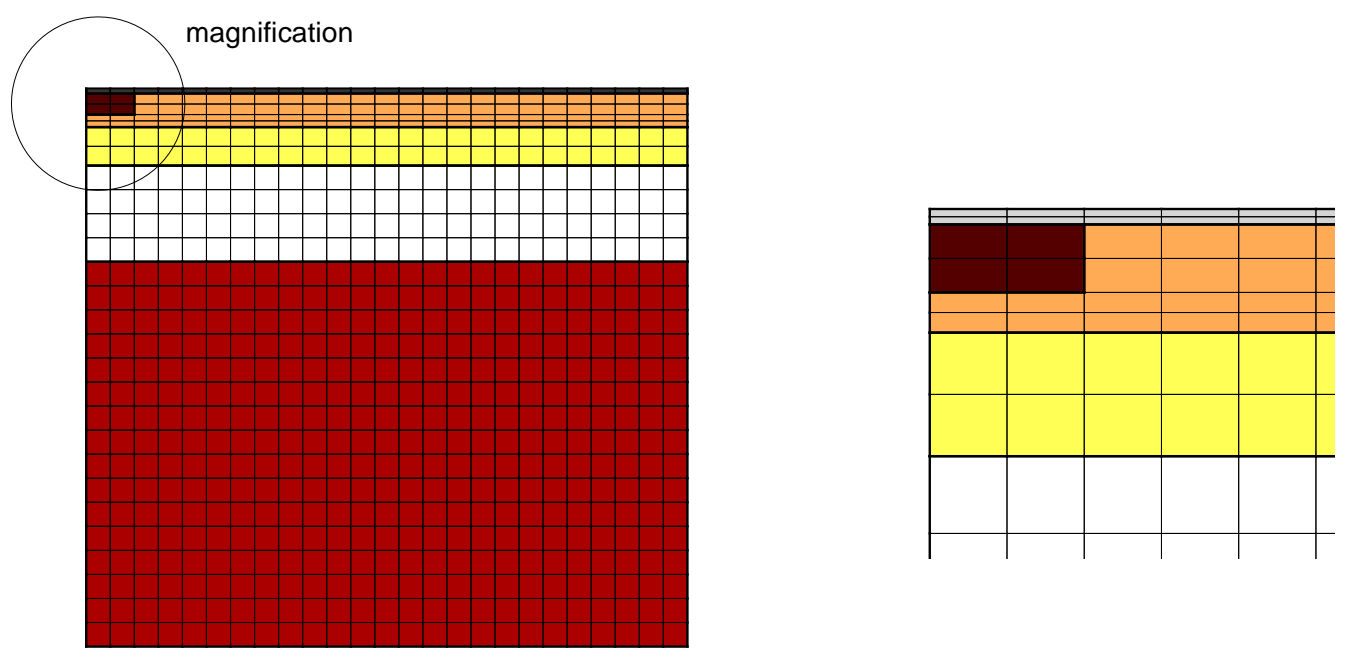

Figure 4: Representative computational mesh for Clark II tumour using an element size of $\Delta r=0.5 \mathrm{~mm}$. 
skin surface temperature, however they did not include in their conclusion the thermal conductivity of fat and muscle. Despite that, they concluded that the variation of the model parameters found in the literature brought a small or negligible surface temperature variation. A model parameter sensitivity analysis has also been done by Cheng and Herman [11] but only for the thickness of the skin layers and their blood perfusion rate. They compare the change in temperature difference between the tumour and healthy skin for a 2D numerical model, changing the parameters randomly. They found that the blood perfusion rate had the highest impact on temperature difference, and that even for a high blood perfusion rate for healthy skin the temperature difference produced by the tumour could still be detected by an IR camera.

This paper offers a more complete sensitivity analysis of 56 model parameters. The reason for this is to detect the most relevant parameters for the numerical modelling of dynamic thermography for skin tumour diagnostic. Looking from a view of direct problems, it is necessary to determine which parameters have the greatest influence on the observed variable and have to be determined more precisely to achieve realistic simulation results. However, when solving inverse problems, it is also necessary to know which parameters have high influence on the problem response, because these parameters can be evaluated based on known experimental data.

The sensitivity analysis has been made based on the gradient of the objective function that can be compared among the parameters, and not on the visual inspection of temperature change as done by Cheng and Herman [11]. Because the most important response in dynamic thermography is the surface temperature difference between the region of the lesion and healthy skin through time, we define the objective function as:

$$
F=\frac{1}{n_{i} n_{t}} \sum_{i=1}^{n_{i}} \sum_{j=1}^{n_{t}} \Delta T_{i, j},
$$

where $n_{i}$ represents the number of spatial points, $n_{t}$ the number of time samples and $\Delta T_{i, j}$ the difference between temperature at spatial points on the skin surface and at a fixed point $(D / 2, H)$ on the healthy skin at certain times, defined as:

$$
\Delta T_{i, j}=T\left(r_{i}, H, t_{j}\right)-T\left(D / 2, H, t_{j}\right) .
$$

We could also choose a different objective function like the time average of the maximal temperature difference or the average value of absolute temperature [10], but the first objective function would lack the spatial information that is also important to reflect the size of the tumour and surrounding tissue properties, and the latter can lead to different conclusions not very important to dynamic thermography where usually the temperature difference is observed. Therefore, the objective function represents an average time and spatial temperature difference during the rewarming period. To evaluate the objective function $F$ for each problem, we calculated the temperature difference for $n_{t}=600$ time steps during the rewarming period, observing the temperature response every $1 s$ and for $n_{i}=11$ equally spaced radial points in the range $r_{i} \in\left[0,2 d_{t}\right]$. We could increase the range, however, the temperature difference after the radius $2 d_{t}$ is close to zero and therefore negligible, and also the number of radial points is sufficient to observe the spatial distribution of temperature difference with the radial step of $0.4 \mathrm{~mm}$ for a Clark II tumour, which is sufficiently small.

To be able to compare the sensitivity of the model parameters we calculated the gradient of the objective function, which represents the relative change of the average spatial 
and time temperature difference with respect to the parameter change. Because we are comparing 56 different model parameters (material properties, geometry and boundary conditions), we define the gradient based on the relative change of the parameter of interest. Therefore, the sensitivity or gradient for each parameter is computed as:

$$
S_{p}=\left|\frac{\partial F}{\partial y_{p}}\right| y_{p} \approx \frac{\left|F\left(y_{p}+\Delta y_{p}\right)-F\left(y_{p}-\Delta y_{p}\right)\right|}{2 \Delta y_{p} / y_{p}},
$$

where $y_{p}$ represents the parameter of interest and $\Delta y_{p}$ its change. As can be seen from equation (32), we used a central FD scheme of second order to evaluate the derivative of the objective function. To evaluate the parameter sensitivity $S_{p}$ we have to calculate the value of the objective function for two different cases, changing the parameter $y_{p}$ by $\Delta y_{p}$, while other parameters were held constant at their reference values. The parameter change $\Delta y_{p}$ has been taken as $1 \%$ of the reference value; $\Delta y_{p}=0.01 y_{p}$. We also tested the accuracy of the gradient calculation with $10 \%$ and $5 \%$ change, as well as a forward difference scheme, and did not notice any difference in the evaluated sensitivity or conclusion. Therefore, the sensitivity evaluated by equation (32) represents the change of average spatial and time temperature difference by the relative change of the investigated model parameter, where $S_{p}$ is expressed in $K$. Because the relative change has been equal for all parameters, their sensitivity can be compared, which helps to identify the most important parameters. To carry out the sensitivity analysis of 56 model parameters for both computational tests, 224 calculations of direct bioheat problems have to be done.

\section{Results and discussion}

In this section, the results of solving direct bio-heat problems for Clark II and IV tumours using a model with local thermoregulation response are initially shown and also compared with a numerical model using constant blood perfusion rate and metabolic heat generation. The results are shown only for the skin surface temperature difference between the tumour and healthy skin, using contour plots for better visual understanding, as well as time and spatial graphs for more accurate presentation of results. We limit our results to temperature difference because it is the most important parameter in dynamic thermography and is also independent of the actual absolute skin temperature, and therefore more general. It is also convenient for comparing results between different experiments using different cooling temperatures or cooling times. In the second part, the results from the sensitivity analysis are presented. The sensitivity analysis has been done for Clark II and IV tumour sizes and is presented in tables for a clear interpretation of the results. Therefore, the results show which model parameters are the most important for successful modelling of dynamic thermography for skin tumour diagnostic.

\subsection{Results of computational examples}

Figure 5 shows the steady-state temperature difference for Clark II and Clark IV tumours, comparing the results from numerical models using thermoregulation (orange line) and constant material properties (gray line). As can be seen, the temperature difference between models is practically identical for both tumours, which means that thermoregulation modelling for steady-state analysis is not important. The blood perfusion rate 

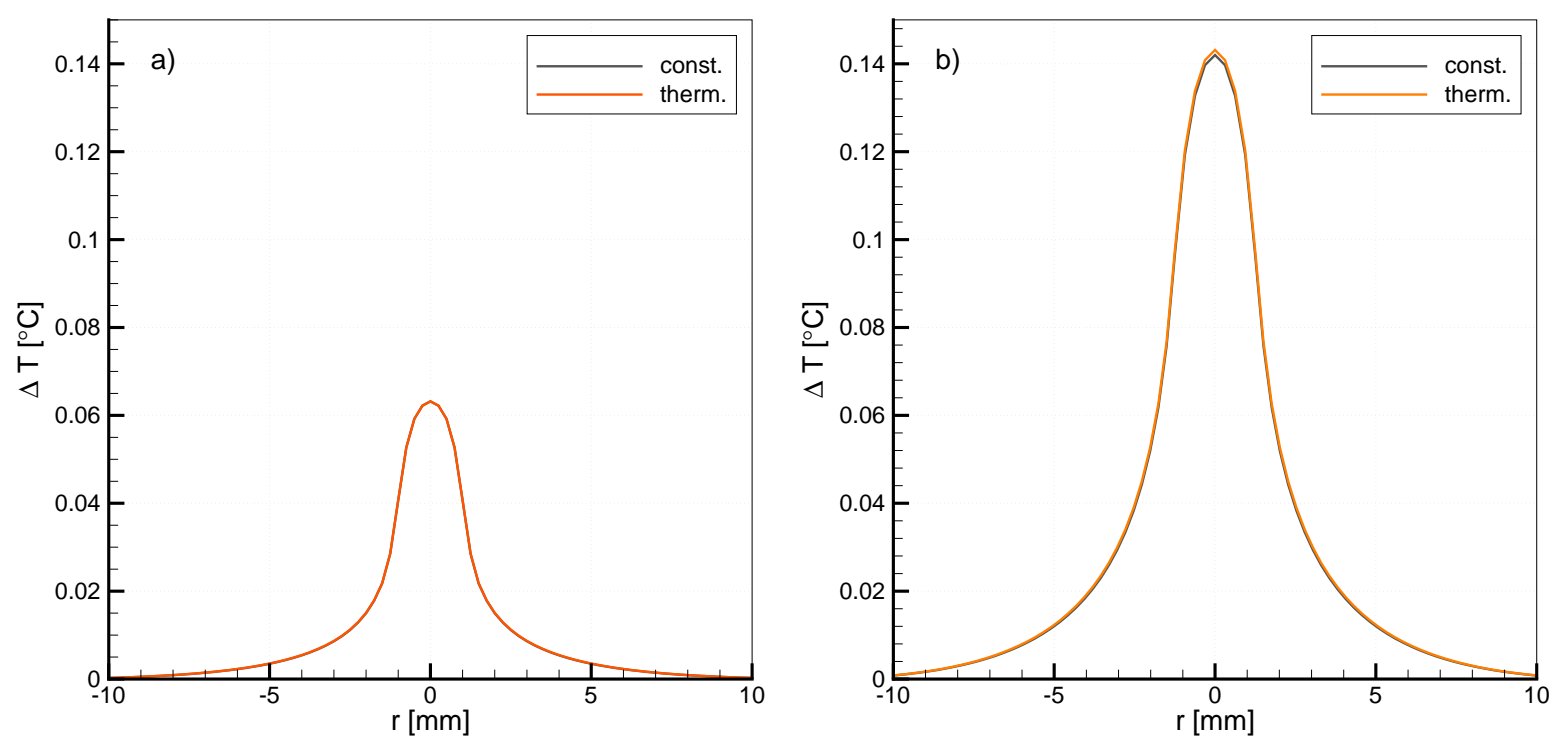

Figure 5: Temperature difference profile on the skin surface under steady-state conditions for: a) Clark II and b) Clark IV tumour.

and metabolic heat generation are equal to their basal value in both cases, which is the reason why the temperature profile is identical. We can also observe that the temperature difference for Clark IV is around $140 \mathrm{mK}$ while for Clark II is $60 \mathrm{mK}$, which is one of the reasons why skin tumours at an early stage are hard to detect using static thermography.

Looking at the temperature response during the rewarming period, shown in Figure 6, we can observe a much higher temperature difference between tumour and healthy skin than in the steady-state case, especially at the beginning of the rewarming period,
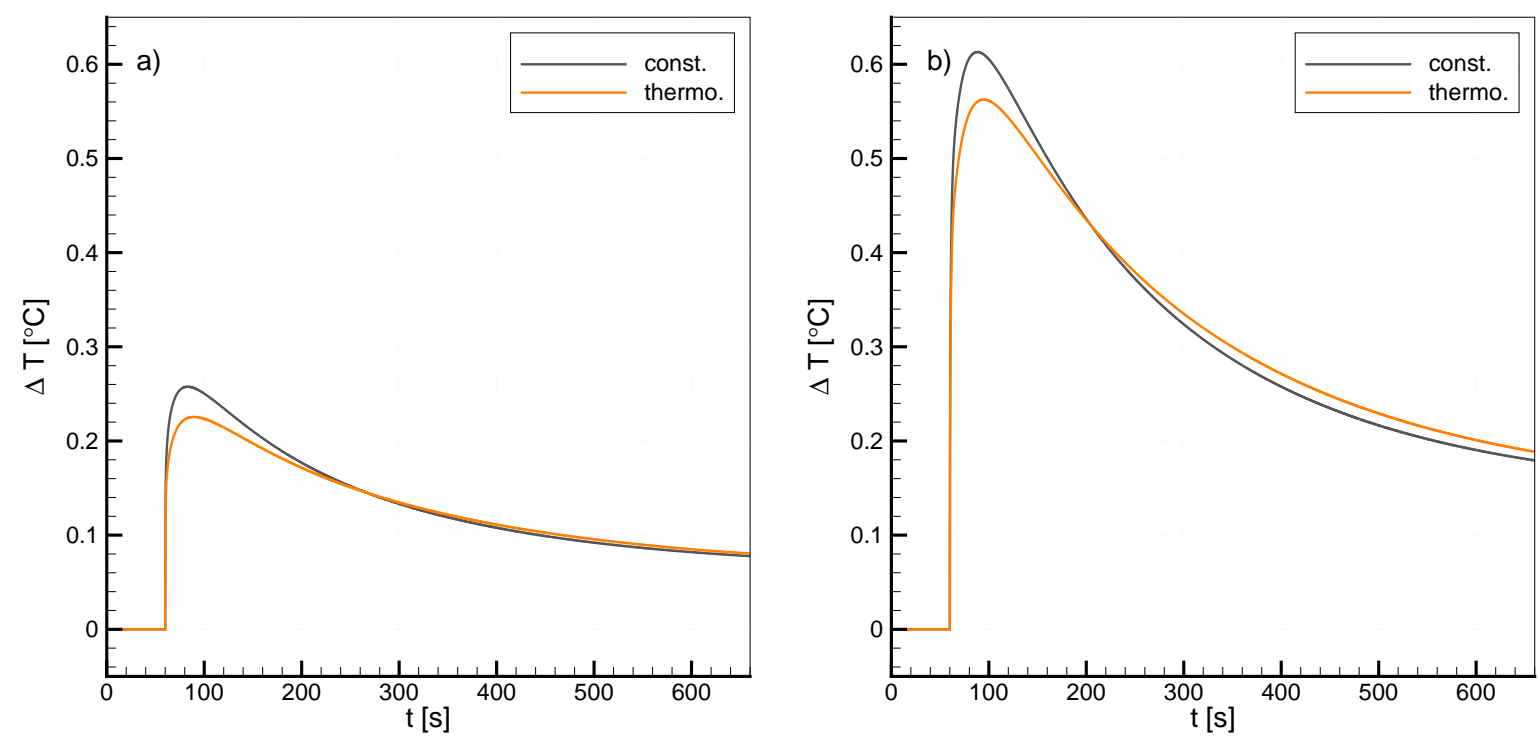

Figure 6: Maximum temperature difference during the rewarming period of dynamic thermography for: a) Clark II and b) Clark IV tumour. 
as has been observed by Çetingül and Herman [14]. Figure 6 shows a maximum temperature difference between tumour centre and surrounding skin, through time, for different numerical models and tumour sizes. In the first $60 \mathrm{~s}$ the skin is cooled to $13^{\circ} \mathrm{C}$ and then exposed to the environment $\left(\alpha=10 \mathrm{~W} / \mathrm{m}^{2} \mathrm{~K}\right.$ and $\left.T_{\infty}=22.4^{\circ} \mathrm{C}\right)$. Comparing the results between the model using constant properties and the thermoregulation model, we can observe that the latter model predicts a lower temperature difference at the peak, and also that the temperature difference decreases through time is slightly slower. This is especially visible for the Clark IV tumour. The predicted peak temperature difference, or the difference between these two models, is around $6.3 \%$ for Clark II and $8.9 \%$ for Clark IV, which is substantial. This indicates that it is necessary to model local thermoregulation response of skin and tumour for realistic modelling of dynamic thermography. The provoked temperature difference in dynamic thermography is now much higher, $200-250 \mathrm{mK}$ for Clark II and $550-600 \mathrm{mK}$ for Clark IV tumour, comparing to the steady-state conditions, and can therefore be more easily detected using an IR camera. It can also be seen that 10min of rewarming period is not enough to obtain a steady-state condition of the skin using the prescribed cooling protocol. Another observation is that even if the temperature peak is missed out, a higher temperature difference can still be detected after $5 \mathrm{~min}$. Of course, the rewarming period is controlled mostly by the amount of cold applied during the cooling phase (cooling temperature and time of cooling), and can thus affect the window time for the rewarming observation [11].

Figures 7 and 8 show the contours of temperature difference at peak time $80 \mathrm{~s}$ for Clark II and Clark IV tumours, respectively. By comparing different numerical models, we can conclude as before that there is an observable difference in peak temperature difference, while the radius of the increased temperature is mostly the same. Therefore, the thermoregulation model will have a noticeable effect on the simulated tissue temperature response and on the estimation of tumour stage or invasiveness, and has to be included in dynamic thermography modelling. The main reason for lower temperature response can be found in the reduced blood perfusion rate of the skin and tumour. However, even if the blood perfusion rate change in skin is higher compared to the tumour during the cooling process, this does not affect the temperature response as much as the change of blood perfusion in the tumour due to a much higher rate. For this reason, the blood perfusion rate of tumour and its thermoregulation response will have a high impact on the simulated temperature response as concluded by the sensitivity analysis.

For visual interpretation of the results using the proposed numerical model, Figures 9 and 10 show contours of temperature difference on the skin surface at different times during the dynamic thermography procedure for Clark II and Clark IV, respectively. Both figures use the same temperature scale to show the difference between the tumour level, where the cut-off level is set to $20 \mathrm{mK}$ [12], which is the current NETD error of IR cameras. The first picture on both figures (part a) represents the steady-state temperature difference and, as already discussed, the temperature signature for the Clark II tumour is almost negligible compared to the Clark IV, and therefore it is harder to detect using static thermography. However, when the skin is cooled down, even an early stage tumour will produce a significant temperature difference during the rewarming period at the peak time that could be more easily detected. The Clark IV tumour will, of course, produce higher temperature differences and is, therefore, not problematic to detect. 


\subsection{Results of sensitivity analysis}

Tables 2 and 3 show the sensitivity calculated by equation (32) for 56 model parameters for Clark II and Clark IV tumours, respectively. The most important parameters for accurate numerical simulation of dynamic thermography are coloured in grey and the intensity of the colour shows the level of importance based on the sensitivity value. We distinguish between important from unimportant parameters based on the maximum value of $S_{p}$ for a given test example. The threshold value has been taken as $10 \%$ of the

a)

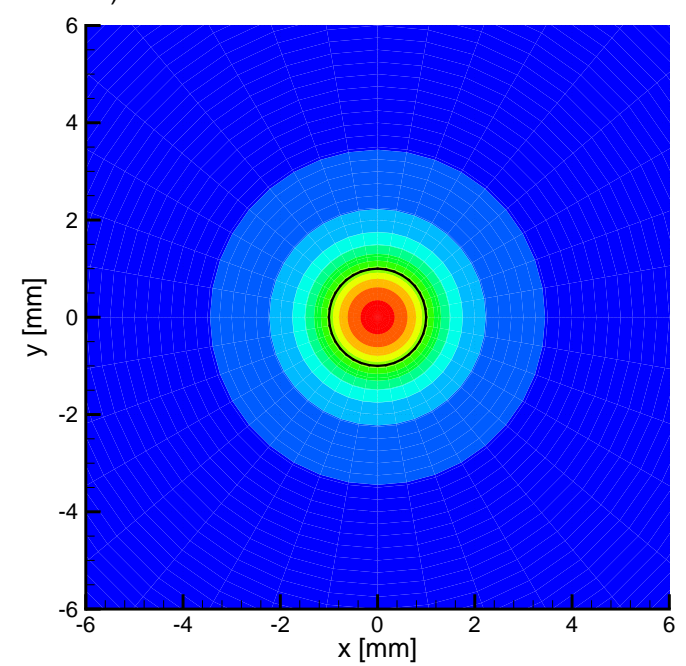

b)

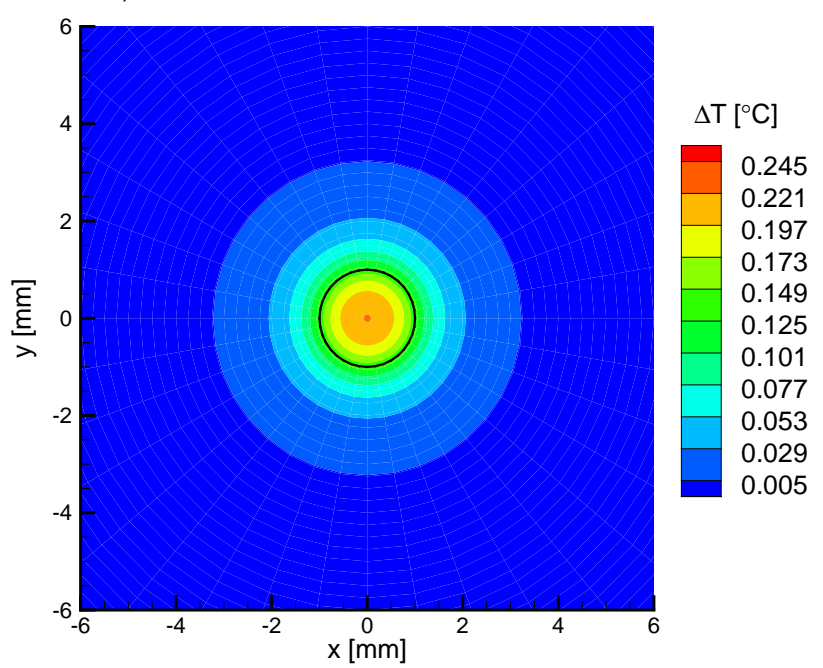

Figure 7: Contours of temperature difference at skin surface for Clark II during the rewarming period of dynamic thermography at $t=80 \mathrm{~s}$ for numerical model using: a) constant properties and b) thermoregulation model.

a)

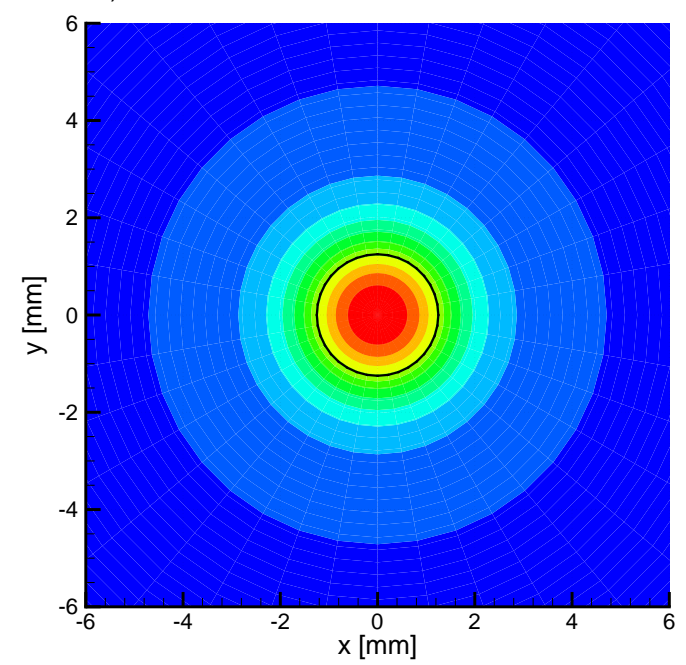

b)

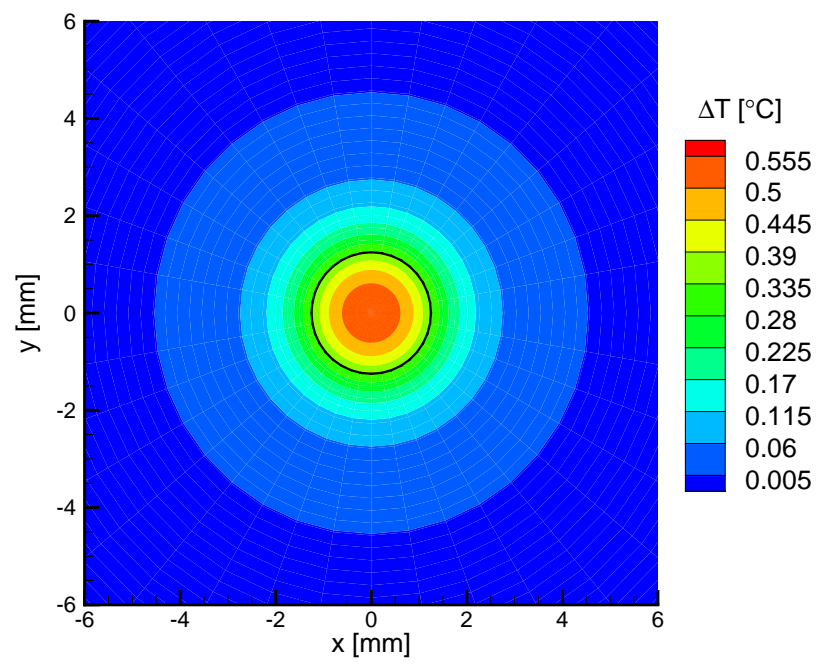

Figure 8: Contours of temperature difference at skin surface for Clark IV during the rewarming period of dynamic thermography at $t=80 \mathrm{~s}$ for numerical model using: a) constant properties and b) thermoregulation model. 
a)

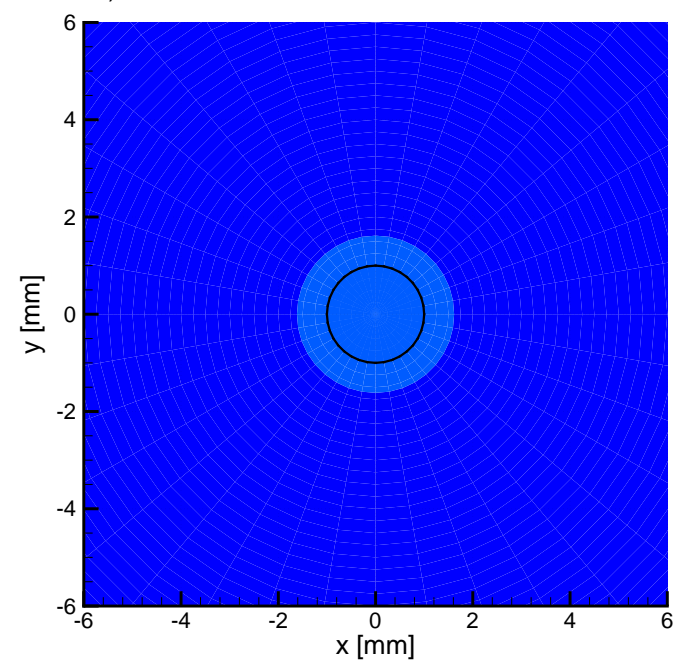

c)

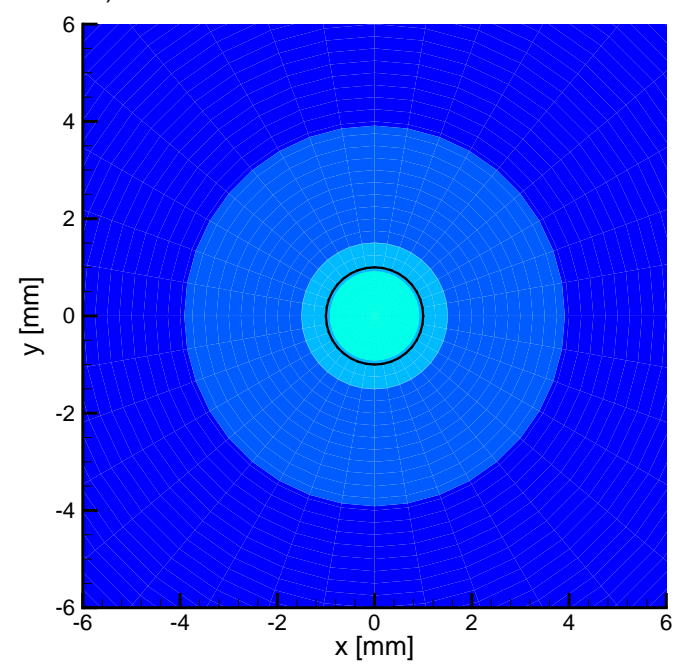

b)

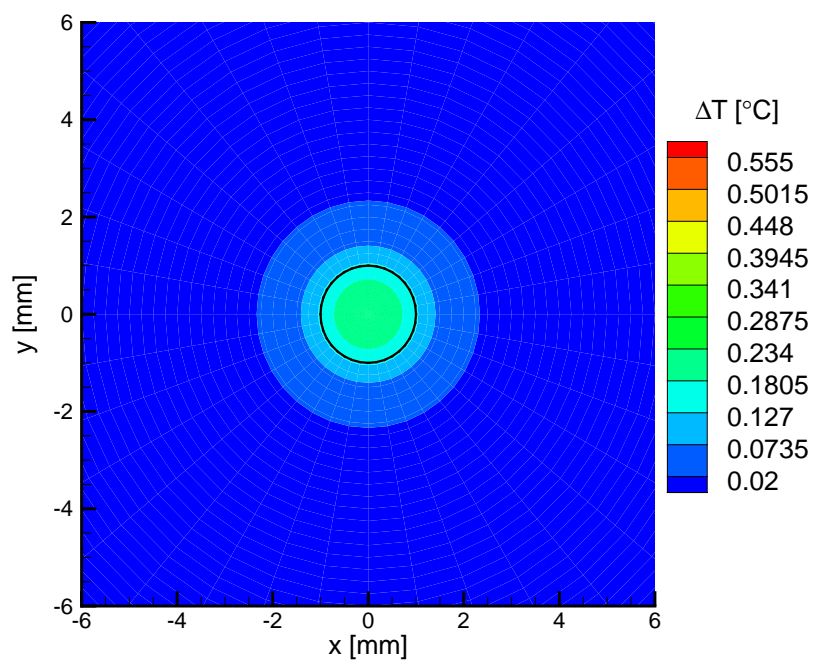

d)

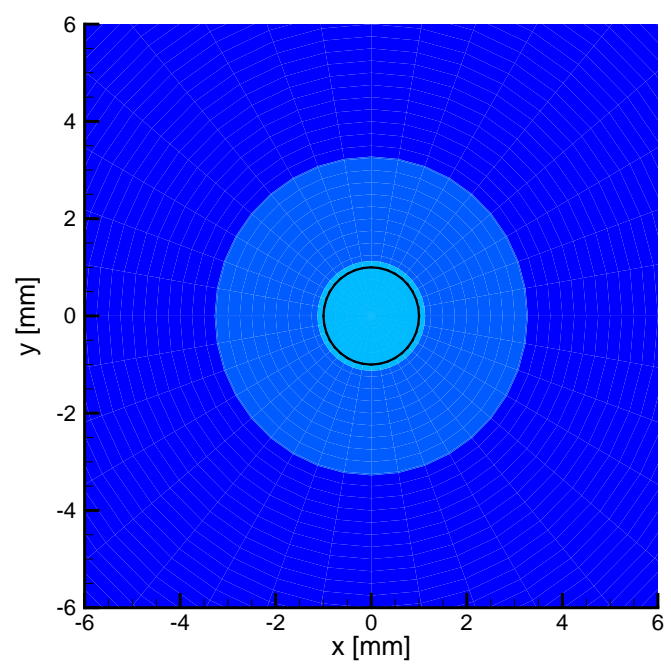

Figure 9: Contours of temperature difference at skin surface for Clark II during the dynamic thermography procedure at different times: a) $t=0 \mathrm{~s}, \mathrm{~b}) t=80 \mathrm{~s}, \mathrm{c}) t=200 \mathrm{~s}$ and d) $t=350 \mathrm{~s}$.

maximum value. This means that the change of average temperature difference will be ten times smaller for unimportant parameters compared to the important ones, which were selected according to the gradient level that is around $50 \%$ of the maximum value or higher, while less important parameters have a lower gradient. From a practical point of view, the values of $S_{p}$ given in Tables 2 and 3 represent the change of average temperature difference in $m K$ if the parameter uncertainty would be $100 \%$, while the change of maximum peak temperature during the rewarming period would be even greater.

As can be seen from Table 2 for Clark II, the most important parameters are density and specific heat of papillary dermis, tumour and blood, blood perfusion rate of tumour, its dimension and thermoregulation coefficient, while for the boundary conditions the body core temperature plays the most important role. However, the cooling temperature, ambient temperature, cooling time, fat thickness and thermal conductivity of papillary 
a)

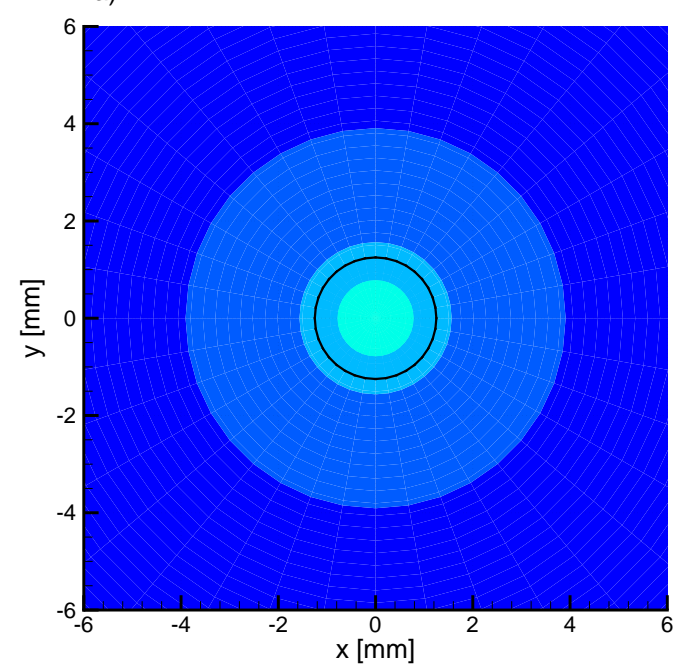

c)

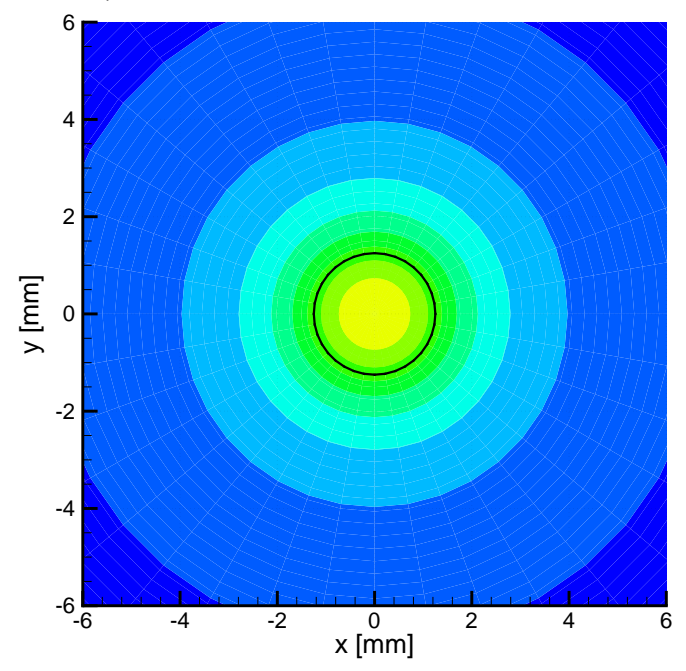

b)

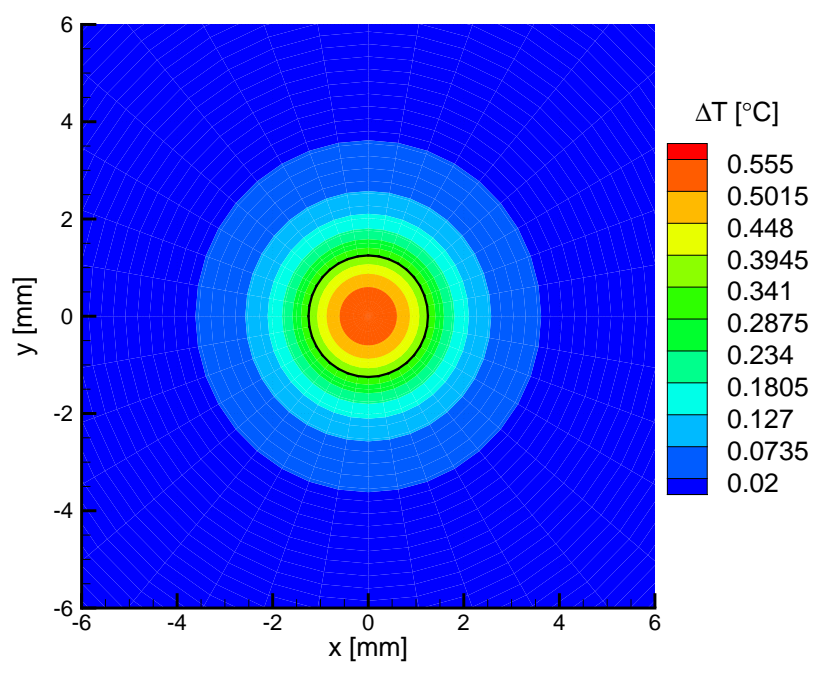

d)

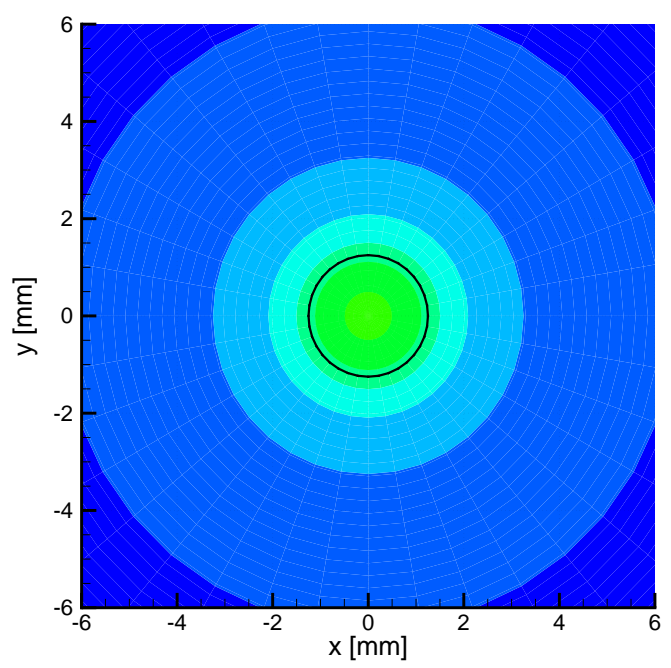

Figure 10: Contours of temperature difference at skin surface for Clark IV during the dynamic thermography procedure at different times: a) $t=0 \mathrm{~s}, \mathrm{~b}) t=80 \mathrm{~s}, \mathrm{c}) t=200 \mathrm{~s}$ and d) $t=350 \mathrm{~s}$.

dermis, reticular dermis, fat and muscle are also not negligible. The value of the sensitivity coefficients is different for Clark II and Clark IV tumours as seen in Tables 2 and 3, where the value for the latter is around two times higher. This just means that the temperature difference change will be higher for Clark IV than for Clark II using the same percentage of parameter change. The significance of each parameter has been chosen for each problem separately, however the same important parameters have been found.

The sensitivity of the metabolic heat generation is around 1000 smaller than the tumour blood perfusion rate, which means that it can be estimated according to literature data and would not affect the results. Because of that, the thermoregulation effect of the metabolic rate does not have great impact and can be neglected. The blood perfusion rate of other layers, except tumour, also does not contribute much because the rate is much smaller than for the tumour, and for this reason the thermoregulation effect in these layers 


\begin{tabular}{c|ccccc|cc|cc} 
material $\backslash$ parameter & $\rho$ & $c_{p}$ & $\lambda$ & $q_{m, \text { bas }}$ & $\omega_{b, \text { bas }}$ & $h$ & $d$ & $Q_{10 m}$ & $Q_{10, b}$ \\
\hline \hline epidermis & 1.75 & 1.75 & 0.63 & 0.0 & 0.0 & 1.21 & - & 0.0 & 0.0 \\
papillary dermis & 43.0 & 43.0 & 25.06 & 0.07 & 1.14 & 0.72 & - & 0.05 & 1.02 \\
reticular dermis & 5.0 & 5.0 & 10.73 & 0.01 & 1.50 & 0.53 & - & 0.01 & 1.56 \\
fat & 1.39 & 1.39 & 18.69 & 0.03 & 0.18 & 15.56 & - & 0.02 & 0.15 \\
muscle & 1.97 & 1.97 & 11.68 & 0.09 & 2.97 & 5.34 & - & 0.02 & 1.01 \\
tumour & 35.56 & 35.56 & 4.62 & 0.94 & 48.58 & 44.99 & 83.77 & 0.71 & 47.64 \\
blood & 42.78 & 42.78 & - & - & - & - & - & - & - \\
\hline bound. cond. parameter & $\alpha$ & $T_{\infty}$ & $T_{0}=T_{a}$ & $T_{c}$ & $t_{c}$ & & & &
\end{tabular}

Table 2: Sensitivity of materials, geometrical, thermoregulation and boundary condition parameters in $m K$ for Clark II numerical model.

\begin{tabular}{c|ccccc|cc|cc} 
material $\backslash$ parameter & $\rho$ & $c_{p}$ & $\lambda$ & $q_{m, \text { bas }}$ & $\omega_{b, \text { bas }}$ & $h$ & $d$ & $Q_{10 m}$ & $Q_{10, b}$ \\
\hline \hline epidermis & 4.18 & 4.18 & 0.62 & 0.0 & 0.0 & 2.28 & - & 0.0 & 0.0 \\
papillary dermis & 84.14 & 84.14 & 40.42 & 0.14 & 2.28 & 7.87 & - & 0.11 & 2.08 \\
reticular dermis & 39.58 & 39.58 & 38.42 & 0.08 & 8.94 & 3.57 & - & 0.06 & 8.48 \\
fat & 3.31 & 3.31 & 54.94 & 0.08 & 0.49 & 44.15 & - & 0.05 & 0.40 \\
muscle & 5.46 & 5.46 & 32.05 & 0.22 & 7.81 & 13.95 & - & 0.05 & 2.70 \\
tumour & 88.74 & 88.74 & 10.75 & 2.48 & 126.1 & 96.75 & 216.9 & 1.84 & 122.4 \\
blood & 106.6 & 106.6 & - & - & - & - & - & - & - \\
\hline bound. cond. parameter & $\alpha$ & $T_{\infty}$ & $T_{0}=T_{a}$ & $T_{c}$ & $t_{c}$ & & & &
\end{tabular}

Table 3: Sensitivity of materials, geometrical, thermoregulation and boundary condition parameters in $m K$ for Clark IV numerical model.

does not play an important role. An important parameter is the blood perfusion rate of tumour and its change due to thermoregulation, and for this it has to be determined more precisely. Cheng and Herman [11] and Çetingül and Herman [14] also changed the first estimated value of the blood perfusion rate of tumour from $0.0064 \mathrm{~s}^{-1}$ to $0.0315 \mathrm{~s}^{-1}$ and $0.045 s^{-1}$ to get results closer to the experimental data.

We also found that density and specific heat capacity of blood play an important role. The reason is due to these two parameters being located in the blood perfusion heat source term of governing equation (1), affecting the rewarming rate similarly to the arterial blood temperature (body core temperature), which is also an important parameter as can be seen from Tables 2 and 3. However, these parameters are well defined or can be easily measured and for that do not present an uncertainty in the numerical model. Similarly, the boundary conditions also have a high impact on the temperature response, but they can be easily measured and defined. It is interesting that the ambient temperature has a greater impact than the heat transfer coefficient meaning that the latter can be estimated. This is convenient because it is hard to determine the value of the heat transfer coefficient exactly during testing. 
The density and specific heat capacity of papillary dermis and tumour are also important parameters. The reason for that can be found in the cooling and rewarming process. The density and specific heat capacity are present in the accumulation term of the governing equation (1) and therefore affect the temperature change through time. For a Clark II tumour, which is embedded in the papillary dermis, these parameters affect the rewarming temperature response, while for a Clark IV tumour, which is also embedded in the reticular dermis, the density and specific heat capacity of this layer also play an important role as can be seen in Table 3, while the epidermis is too thin to have any effect at all.

The thermal conductivity of the different layers, which play an important role in heat transfer during the cooling-rewarming period, also have some influence on the average temperature difference. This parameter mostly affects the cooling period and determines the cooling penetration depth, which is also controlled by the cooling temperature and cooling time, as can be seen in Tables 2 and 3 that consequently affects the rewarming speed and therefore the observed temperature difference. However, the sensitivity is around $3-5$ times smaller than that for the blood perfusion rate of the tumour.

Looking at the geometry of the model or layer thickness, we can see that the highest influence comes from the tumour thickness and diameter, which describe the tumour volume, when other thicknesses are not so important, especially the skin thickness. The thickness of the fat layer also has some effect on the temperature difference during the rewarming period, as the fat acts like insulation and reduces the cooling penetration depth.

To conclude, the most important parameters that control the simulated temperature difference between tumour and healthy skin during the rewarming period of dynamic thermography, and are difficult to be exactly measured for individual patients and represent model uncertainty, are density and specific heat capacity of tumour and the skin layer in which the tumour is embedded, volume of the tumour, its blood perfusion rate and thermoregulation change. Other important parameters are well defined or can be easily measured and controlled, while not so important parameters can be estimated using average values found in the literature.

\section{Conclusion}

Dynamic thermography has been shown in many papers to be a promising new noninvasive diagnostic approach not only for skin tumour but also for other applications in medicine like breast cancer detection, etc. Realistic and accurate numerical simulation of bioheat transfer in tissue during dynamic thermography can increase the application of this technique, however, for this to happen, we need to improve existing models to describe the simulated phenomena correctly.

The paper presents a numerical model of a multilayer skin tumour tissue based on the Pennes bioheat equation and thermoregulation response of the skin and tumour to simulate the transient thermal response of the tissue during dynamic thermography more realistically. The thermoregulation response is modelled by temperature-dependent metabolic heat generation and blood perfusion rate using exponential behaviour. The non-linear bioheat problem has been treated as 3D-axisymmetrical due to the cylindrical shape of the tumour and computational domain and solved using an efficient subdomain BEM 
approach.

We solved two examples for Clark II and Clark IV tumours to present the advantages of dynamic thermography for skin tumour detection, as well as the difference between numerical models using constant material properties or local thermoregulation response for blood perfusion rate and metabolic heat generation. The results show that including a thermoregulation response of the tissue affects the simulated temperature response during the rewarming period of dynamic thermography, especially for the temperature difference peak, and has to be considered for accurate and realistic modelling. In these examples, the error produced by constant material properties, not including the thermoregulation response, can be in the range of $6 \%$ for Clark II and $9 \%$ for Clark IV, which is substantial if using the model for solving inverse bioheat problems to estimate tumour geometrical and physiological parameters.

One of the contributions of this paper is also a detailed sensitivity analysis of the model parameters, which has been done by calculating the gradient of the objective function. For this problem, we define the objective function as a spatial and time average of the observed temperature difference at the skin surface during the rewarming period. The analysis has been done for 56 model parameters, which showed the most important parameters that affect the simulated temperature response. These parameters have to be determined more precisely if we wish to simulate dynamic thermography accurately. Among the 56 parameters, the most important are blood perfusion rate and its thermoregulation response for tumour, density and specific heat capacity of blood, tumour and tissue layers in which the tumour is embedded, size of tumour and body core or arterial blood temperature. Some of these parameters can be determined or measured quite easily, while others need a more detailed investigation. On the other hand, the metabolic heat generation showed very low sensitivity meaning that it can be estimated from the literature, and so there is no need to model the thermoregulation response of this parameter. The second most important parameters are the fat thickness, thermal conductivity of layers, ambient temperature and cooling protocol.

The paper presents theoretical work and two novelties in the field of numerical modelling of dynamic thermography for skin tumour diagnostic. The first one is an improved 3D numerical multilayer model that includes the thermoregulation response of the tissue and tumour, while the second is a complete sensitivity analysis of the model parameters, which will contribute to further developments in the field of bioheat modelling and solving inverse problems in dynamic thermography. The numerical model presented in this paper could also be further improved by including lesions of non-cylindrical shapes, thermal contact resistance between the cooling element and skin surface, raised lesions that contribute to non-symmetrical thermal contrast during the recovery phase.

\section{References}

[1] M. Diakides, J. D. Bronzino, D. R. Peterson, Medical Infrared Imaging: Principles and Practices, CRC Press, 2012.

[2] M. Bonmarin, L. G. Frédérique-Anne, Thermal imaging in dermatology, in: Imaging in Dermatology, Academic Press, 2016, pp. 437-454.

[3] B. Lahiri, S. Bagavathiappan, T. Jayakumar, J. Philip, Medical applications of in- 
frared thermography: A review, Infrared Physics \& Technology 55 (4) (2012) 221235.

[4] C. Magalhaes, R. Vardasca, J. Mendes, Recent use of medical infrared thermography in skin neoplasms, Skin Research and Technology 24 (4) (2018) 587-591.

[5] A. Di Carlo, Thermography and the possibilities for its applications in clinical and experimental dermatology, Clinics in Dermatology 13 (4) (1995) 329-336.

[6] M. Bonmarin, F.-A. Le Gal, A lock-in thermal imaging setup for dermatological applications, Skin Research and Technology 21 (3) (2015) 284-290.

[7] M. Bonmarin, F.-A. Le Gal, Lock-in thermal imaging for the early-stage detection of cutaneous melanoma: A feasibility study, Computers in Biology and Medicine 47 (2014) 36-43.

[8] S. E. Godoy, D. A. Ramirez, S. A. Myers, G. von Winckel, S. Krishna, M. Berwick, R. S. Padilla, P. Sen, S. Krishna, Dynamic infrared imaging for skin cancer screening, Infrared Physics \& Technology 70 (2015) 147-152.

[9] C. Herman, The role of dynamic infrared imaging in melanoma diagnosis, Expert Review of Dermatology 8 (2) (2013) 177-184.

[10] M. Çetingül, C. Herman, A heat transfer model of skin tissue for detection of lesions: Sensitivity analysis, Physics in Medicine and Biology 55 (2010) 5933-5951.

[11] T.-Y. Cheng, C. Herman, Analysis of skin cooling for quantitative dynamic infrared imaging of near-surface lesions, International Journal of Thermal Sciences 86 (2014) $175-188$.

[12] M. Stra̧kowska, R. Stra̧kowski, M. Strzelecki, G. De Mey, B. Więcek, Evaluation of perfusion and thermal parameters of skin tissue using cold provocation and thermographic measurements, Metrology and Measurement Systems 23 (3) (2016) 373-381.

[13] A. Amri, S. H. Pulko, A. J. Wilkinson, Potentialities of steady-state and transient thermography in breast tumour depth detection: A numerical study, Computer Methods and Programs in Biomedicine 123 (2016) 68-80.

[14] M. P. Çetingül, C. Herman, Quantification of the thermal signature of a melanoma lesion, International Journal of Thermal Sciences 50 (4) (2011) 421-431.

[15] W. H. Clark, L. From, E. A. Bernardino, M. C. Mihm, The histogenesis and biologic behavior of primary human malignant melanomas of the skin, Cancer Research 29 (3) (1969) 705-727.

[16] A. A. Marghoob, K. Koenig, F. V. Bittencourt, A. W. Kopf, R. S. Bart, Breslow thickness and Clark level in melanoma: support for including level in pathology reports and in American Joint Committee on Cancer staging, Cancer 88 (3) (2000) $589-595$. 
[17] J. K. Patel, S. Konda, O. A. Perez, S. Amini, G. Elgart, B. Berman, Newer technologies/techniques and tools in the diagnosis of melanoma, European Journal of Dermatology 18 (6) (2008) 617-631.

[18] D. S. Rigel, J. Russak, R. Friedman, The evolution of melanoma diagnosis: 25 years beyond the ABCDs, CA: A Cancer Journal for Clinicians 60 (5) (2010) 301-316.

[19] S. E. Godoy, M. M. Hayat, D. A. Ramirez, S. A. Myers, R. S. Padilla, S. Krishna, Detection theory for accurate and non-invasive skin cancer diagnosis using dynamic thermal imaging, Biomedical Optics Express 8 (4) (2017) 2301-2323.

[20] M. Stücker, I. Horstmann, C. Nüchel, A. Röchling, K. Hoffmann, P. Altmeyer, Blood flow compared in benign melanocytic naevi, malignant melanomas and basal cell carcinomas, Clinical and Experimental Dermatology 24 (2) (1999) 107-111.

[21] C. W. Song, A. Lokshina, J. G. Rhee, M. Patten, S. H. Levitt, Implication of blood flow in hyperthermic treatment of tumors, IEEE Transactions on Biomedical Engineering 31 (1) (1984) 9-16.

[22] C. W. Song, J. G. Rhee, S. H. Levitt, Blood flow in normal tissues and tumors during hyperthermia, Journal of the National Cancer Institute 64 (1) (1980) 119-124.

[23] C. Laurino, B. Palmieri, Wide instrumental screening in monitoring early melanoma, European Journal of Oncology 20 (1) (2015) 41-52.

[24] K. Ammer, E. Ring, Standard Procedures for Infrared Imaging in Medicine, Biomedical Engineering Handbook, CRC Press, 2006.

[25] A. R. Melo, M. M. S. Loureiro, F. Loureiro, Blood perfusion parameter estimation in tumors by means of a genetic algorithm, Procedia Computer Science 108 (2017) $1384-1393$.

[26] P. W. Partridge, L. C. Wrobel, An inverse geometry problem for the localisation of skin tumours by thermal analysis, Engineering Analysis with Boundary Elements 31 (10) (2007) 803-811.

[27] P. W. Partridge, L. C. Wrobel, A coupled dual reciprocity BEM/genetic algorithm for identification of blood perfusion parameters, International Journal of Numerical Methods for Heat \& Fluid Flow 19 (1) (2009) 25-38.

[28] J. M. Luna, R. Romero-Mendez, A. Hernandez-Guerrero, F. Elizalde-Blancas, Procedure to estimate thermophysical and geometrical parameters of embedded cancerous lesions using thermography, Journal of Biomechanical Engineering 134 (3) (2012) 031008-1-031008-9.

[29] J. M. Luna, A. Hernández Guerrero, R. Romero Méndez, J. L. Luviano Ortiz, Solution of the inverse bio-heat transfer problem for a simplified dermatological application: Case of skin cancer, Ingeniería Mecánica, Tecnología y Desarrollo 4 (6) (2014) $219-228$. 
[30] M. Paruch, E. Majchrzak, Identification of tumor region parameters using evolutionary algorithm and multiple reciprocity boundary element method, Engineering Applications of Artificial Intelligence 20 (5) (2007) 647-655.

[31] L. A. Dombrovsky, V. Timchenko, C. Pathak, H. Piazena, W. Müller, M. Jackson, Radiative heating of superficial human tissues with the use of water-filtered infraredA radiation: A computational modeling, International Journal of Heat and Mass Transfer 85 (2015) 311-320.

[32] A. Bhowmik, R. Repaka, S. C. Mishra, Thermographic evaluation of early melanoma within the vascularized skin using combined non-Newtonian blood flow and bioheat models, Computers in Biology and Medicine 53 (2014) 206-219.

[33] A. Bhowmik, R. Repaka, R. Mulaveesala, S. C. Mishra, Suitability of frequency modulated thermal wave imaging for skin cancer detection - A theoretical prediction, Journal of Thermal Biology 51 (2015) 65-82.

[34] A. Bhowmik, R. Repaka, Estimation of growth features and thermophysical properties of melanoma within 3-D human skin using genetic algorithm and simulated annealing, International Journal of Heat and Mass Transfer 98 (2016) 81-95.

[35] D. Fiala, G. Havenith, P. Bröde, B. Kampmann, G. Jendritzky, UTCI-Fiala multinode model of human heat transfer and temperature regulation, International Journal of Biometeorology 56 (3) (2012) 429-441.

[36] I. Laakso, A. Hirata, Dominant factors affecting temperature rise in simulations of human thermoregulation during RF exposure, Physics in Medicine \& Biology 56 (23) (2011) 7449 .

[37] V. Vuksanović, L. W. Sheppard, A. Stefanovska, Nonlinear relationship between level of blood flow and skin temperature for different dynamics of temperature change, Biophysical Journal 94 (10) (2008) L78-L80.

[38] A. B. C. Silva, J. Laszczyk, L. C. Wrobel, F. L. Ribeiro, A. J. Nowak, A thermoregulation model for hypothermic treatment of neonates, Medical Engineering \& Physics 38 (9) (2016) 988-998.

[39] C. B. Pereira, K. Heimann, M. Czaplik, V. Blazek, B. Venema, S. Leonhardt, Thermoregulation in premature infants: A mathematical model, Journal of Thermal Biology 62 (2016) 159-169.

[40] J. E. Laszczyk, A. J. Nowak, Computational modelling of neonate's brain cooling, International Journal of Numerical Methods for Heat \& Fluid Flow 26 (2) (2016) $571-590$.

[41] A. Bhowmik, R. Singh, R. Repaka, S. C. Mishra, Conventional and newly developed bioheat transport models in vascularized tissues: A review, Journal of Thermal Biology 38 (3) (2013) 107-125.

[42] F. Xu, K. Seffen, T. Lu, Non-Fourier analysis of skin biothermomechanics, International Journal of Heat and Mass Transfer 51 (9) (2008) 2237-2259. 
[43] A. Nakayama, F. Kuwahara, A general bioheat transfer model based on the theory of porous media, International Journal of Heat and Mass Transfer 51 (11) (2008) 3190-3199.

[44] H. H. Pennes, Analysis of tissue and arterial blood temperatures in the resting human forearm, Journal of Applied Physiology 1 (2) (1948) 93-122.

[45] S. B. Wilson, V. A. Spence, A tissue heat transfer model for relating dynamic skin temperature changes to physiological parameters, Physics in Medicine \& Biology 33 (8) (1988) 895-912.

[46] J. Werner, M. Buse, Temperature profiles with respect to inhomogeneity and geometry of the human body, Journal of Applied Physiology 65 (3) (1988) 1110-1118.

[47] D. Fiala, K. J. Lomas, M. Stohrer, A computer model of human thermoregulation for a wide range of environmental conditions: the passive system, Journal of Applied Physiology 87 (5) (1999) 1957-1972.

[48] J. W. Mitchell, T. L. Galvez, J. Hengle, G. E. Myers, K. L. Siebecker, Thermal response of human legs during cooling, Journal of Applied Physiology 29 (6) (1970) 859-865.

[49] J. A. Stolwijk, A mathematical model of physiological temperature regulation in man, NASA contractor report, NASA CR-1855, 1971.

[50] C. K. Charny, M. J. Hagmann, R. L. Lavin, A whole body thermal model of man during hyperthermia, IEEE Transactions on Biomedical Engineering (5) (1987) 375387.

[51] S. Kikuchi, K. Saito, M. Takahashi, K. Ito, Temperature elevation in the fetus from electromagnetic exposure during magnetic resonance imaging, Physics in Medicine \& Biology 55 (8) (2010) 2411-2426.

[52] P. Bernardi, M. Cavagnaro, S. Pisa, E. Piuzzi, Specific absorption rate and temperature elevation in a subject exposed in the far-field of radio-frequency sources operating in the 10-900-MHz range, IEEE Transactions on Biomedical Engineering 50 (3) (2003) 295-304.

[53] D. Nelson, S. Charbonnel, A. Curran, E. Marttila, D. Fiala, P. Mason, J. Ziriax, A high-resolution voxel model for predicting local tissue temperatures in humans subjected to warm and hot environments, Journal of Biomechanical Engineering 131 (4) (2009) 041003-1-12.

[54] Y. Shen, P. Liu, A. Zhang, L. X. Xu, Tumor microvasculature response to alternated cold and heat treatment, in: 2005 IEEE Engineering in Medicine and Biology 27th Annual Conference, IEEE, 2006, pp. 6797-6800.

[55] J. Nagy, S. Chang, A. Dvorak, H. Dvorak, Why are tumour blood vessels abnormal and why is it important to know?, British Journal of Cancer 100 (6) (2009) 865-869. 
[56] J. Lang, B. Erdmann, M. Seebass, Impact of nonlinear heat transfer on temperature control in regional hyperthermia, Transactions on Biomedical Engineering 46 (9) (1999) 1129-1138.

[57] T. Drizdal, P. Togni, L. Visek, J. Vrba, Comparison of constant and temperature dependent blood perfusion in temperature prediction for superficial hyperthermia, Radioengineering 19 (2) (2010) 281-289.

[58] J. Sun, A. Zhang, L. X. Xu, Evaluation of alternate cooling and heating for tumor treatment, International Journal of Heat and Mass Transfer 51 (23-24) (2008) 54785485 .

[59] E. R. Nadel, R. W. Bullard, J. Stolwijk, Importance of skin temperature in the regulation of sweating., Journal of Applied Physiology 31 (1) (1971) 80-87.

[60] I. B. Mekjavić, J. Bligh, Core threshold temperatures for sweating, Canadian Journal of Physiology and Pharmacology 67 (9) (1989) 1038-1044.

[61] M. Ramšak, L. Škerget, A highly efficient multidomain BEM for multimillion subdomains, Engineering Analysis with Boundary Elements 43 (2014) 76 - 85.

[62] M. Ramšak, L. Škerget, Heat diffusion in fractal geometry cooling surface, Thermal Science 16 (4) (2012) 955-968.

[63] J. Ravnik, L. Škerget, Z. Žunič, Velocity-vorticity formulation for 3D natural convection in an inclined enclosure by BEM, International Journal of Heat and Mass Transfer 51 (17) (2008) 4517-4527.

[64] J. Ravnik, M. Hriberšek, J. Lupše, Lagrangian particle tracking in velocity-vorticity resolved viscous flows by subdomain BEM, Journal of Applied Fluid Mechanics 9 (3) (2016) 1533-1549.

[65] J. Iljaž, L. C. Wrobel, M. Hriberšek, J. Marn, Subdomain BEM formulations for the solution of bio-heat problems in biological tissue with melanoma lesions, Engineering Analysis with Boundary Elements 83 (2017) 25-42.

[66] L. C. Wrobel, The Boundary Element Method: Applications in Thermo-Fluids and Acoustics, Vol. 1, John Wiley \& Sons, 2002.

[67] M. Ramšak, J. Ravnik, M. Zadravec, M. Hriberšek, J. Iljaž, Freeze-drying modeling of vial using BEM, Engineering Analysis with Boundary Elements 77 (2017) 145-156.

[68] A. Breslow, Thickness, cross-sectional areas and depth of invasion in the prognosis of cutaneous melanoma, Annals of Surgery 172 (5) (1970) 902-908.

[69] J. Iljaž, L. C. Wrobel, M. Hriberšek, J. Marn, The use of Design of Experiments for steady-state and transient inverse melanoma detection problems, International Journal of Thermal Sciences 135 (2019) 256-275. 